\title{
Bildung und subjektives Wohlbefinden im Zeitverlauf, 1984-2002
}

\author{
Eine Mehrebenenanalyse
}

\author{
Andreas Hadjar · Sigrid Haunberger $\cdot$ Frank Schubert
}

Zusammenfassung: Diese Analyse des subjektiven Wohlbefindens verfolgt zwei Ziele. Zum einen wird die Entwicklung des subjektiven Wohlbefindens über die Periode zwischen 1984 und 2002 untersucht. Dabei werden Alters-, Perioden- und Kohorteneffekte simultan betrachtet. Als soziale Mechanismen hinter den Wandlungsprozessen, das heißt als Prädiktoren für das subjektive Wohlbefinden und die Variation im Zeitverlauf, werden Bildung und Bildungsexpansion thematisiert und in die Analysemodelle eingeführt. Um die Lebenszufriedenheit unabhängig von gesundheitlichen Aspekten zu untersuchen, wird die Gesundheitszufriedenheit kontrolliert. Methodisch folgt der Beitrag einem Mehrebenenansatz. Zur Datenanalyse werden Hierarchisch Lineare Modelle (HLM) herangezogen. Detailliert wird auf die benötigte Datenstruktur, die Anwendbarkeit und die Parameter-Schätzungen von HLM eingegangen. Die Datengrundlage bildet das Sozio-Ökonomische Panel (SOEP), wobei nur die Teil-Stichprobe der Westdeutschen in die Analyse einbezogen wird. In den Resultaten zeigen sich - neben einem starken Einfluss der Gesundheitszufriedenheit - u. a. robuste Bildungseffekte, ein substanzieller Alterseffekt und ein schwacher negativer Kohorteneffekt auf die Lebenszufriedenheit.

Schlüsselwörter: Wohlbefinden $\cdot$ Arbeitslosigkeit $\cdot$ A-P-K-Analyse $\cdot$ Mehrebenenansatz

Education and Subjective Well-Being in Temporal Perspective, 1984-2002: A Multi-Level Analysis

\begin{abstract}
This analysis of the change in subjective well-being attempts two aims. On the one hand, the change of subjective well-being over the time period from 1984 to 2002 will be considered. Effects of age, period and cohorts will be analyzed simultaneously. Education and educational expansion are considered as social mechanisms behind these processes of change and therefore introduced into the analyses. Methodologically, a multilevel approach is chosen. To analyze life satisfaction independently from health aspects, satisfaction with health will be controlled for. Hierarchical Linear Models (HLM) will be used for data analysis. Data structure, applicability
\end{abstract}

Andreas Hadjar $(\bowtie) \cdot$ Sigrid Haunberger $\cdot$ Frank Schubert Abteilung Bildungssoziologie, Universität Bern, Muesmattstraße 27, 3012 Bern, Switzerland

E-Mail: andreas.hadjar@edu.unibe.ch; sigrid.haunberger@edu.unibe.ch; frank.schubert@edu.unibe.ch 
and parameter estimations of HLM will be considered in detail. The data base is the German Socio-economic Panel data set, although only the West German subsample is used for analysis. Results indicate - beside a strong influence of health satisfaction - robust effects of education, a substantial age effect and a weak negative cohort effect on life satisfaction.

Keywords: Well-being $\cdot$ Education $\cdot$ Unemployment $\cdot$ Age-Period-Cohort-Analysis $\cdot$ Multi-level Approach

Résumé: L'analyse du bien-être subjectif poursuit deux objectifs. Nous étudions d'une part l'évolution du bien-être subjectif (satisfaction à l'égard de la vie) sur la période comprise entre 1984 et 2002. Ce faisant, nous prenons simultanément en considération les effets d'âge, de période et de cohorte. L'éducation et la démocratisation de l'éducation sont abordées et intégrées dans les modèles d'analyse comme mécanismes sociaux à l'œuvre derrière les processus de changement, c'est-à-dire comme prédicteurs du bien-être subjectif et de sa variation dans le temps. Pour étudier la satisfaction à l'égard de la vie indépendamment des aspects médicaux, nous contrôlons la satisfaction à l'égard de la santé. Sur le plan méthodologique, cet article adopte une approche multi-niveaux. Pour analyser les données, nous recourons à des modèles linéaires hiérarchiques $(\mathrm{MLH})$, la structure de données requise, l'applicabilité et les estimations de paramètres des MLH étant présentées de façon détaillée. Les données utilisées sont tirées du panel socio-économique (SOEP), l'échantillon partiel des Allemands de l'Ouest étant inclus dans l'analyse. Les résultats révèlent notamment - outre une forte influence de la satisfaction à l'égard de la santé - de solides effets d'éducation, un effet d'âge substantiel et un léger effet de cohorte négatif sur la satisfaction à l'égard de la vie.

Mots-clés: bien-être $\cdot$ chômage $\cdot$ analyse APC $\cdot$ approche multi-niveaux

\section{Einleitung}

Die Frage danach, was subjektives Wohlbefinden ausmacht und wie es erzeugt wird, ist eine Kernfrage der Sozialwissenschaften. Glück und Zufriedenheit mit dem Leben bzw. Teilaspekten des Lebens sind wichtige Einstellungsmuster; das Streben nach subjektivem Wohlbefinden gilt als der Motor sozialen Handelns - und dies auch über das Rational-Choice-Paradigma (Ormel et al. 1999) hinaus. Aber nicht nur aus der individuellen Perspektive ist das subjektive Wohlbefinden von Interesse: Das aggregierte subjektive Wohlbefinden kann ein Indikator für die soziale Integration und die Lebbarkeit einer Gesellschaft sein, ein Maß dafür, inwieweit ihre Angebote, Anforderungen und Entfaltungsmöglichkeiten mit den Bedürfnissen und Fähigkeiten ihrer Bürger korrespondieren (Veenhoven 1997: 268). Die Forschungstraditionen zur Lebensqualität und zum Wohlbefinden haben sich in den letzten Jahren angenähert. Ursprünglich geht die Lebensqualitätsforschung auf die sozialwissenschaftliche Wohlfahrts- und Sozialindikatorenforschung zurück. Mit Lebensqualität wird ein allgemeines Maß der Kongruenz von objektiven Lebensbedingungen und deren subjektiver Bewertung (Wohlbefinden, Zufriedenheit) verstanden (vgl. Noll 1989; Glatzer/Zapf 1984b; Zapf/Schupp/Habich 1996). Bezüglich der Bestimmungsfaktoren subjektiven Wohlbefindens werden u.a. sozialstrukturelle Faktoren, soziale Netzwerke, der Partnerschaftsstatus, Gesundheit sowie psychologische Faktoren wie die Selbstwirksamkeit im wissenschaftlichen Dis- 
kurs thematisiert (Baier/Boehnke 2007). So erfreuen sich einer höheren Sozialschicht angehörende, finanziell gut ausgestattete, in ein soziales Netzwerk eingebundene, in Partnerschaft lebende (Glatzer/Zapf 1984a: 396) Individuen, die über eine hohe Selbstwirksamkeit verfügen, einer hohen Lebenszufriedenheit - denn die Zufriedenheit mit dem Lebensstandard, dem Einkommen, der Familie und der Gesundheit hängen eng mit dem subjektiven Wohlbefinden zusammen (vgl. Spellerberg/Landua/Habich 1992; Glatzer/Zapf 1984b).

Die folgenden Analysen fokussieren als wesentlichen Bestimmungsfaktor des Wohlbefindens das Bildungsniveau - zum einen als kognitive Ressource, zum anderen als Humankapital, das in Status und Einkommen umsetzbar ist. Die Wichtigkeit dieser Erklärungsvariablen ergibt sich daraus, dass sich mit höherer Bildung bessere kognitive und materielle Chancen eröffnen und sich so ein besseres Wohlbefinden herstellen lässt. Die Bildung beeinflusst eine Vielzahl der Bestimmungsfaktoren des subjektiven Wohlbefindens. Um die sozialen Mechanismen adäquat zu untersuchen, werden Bildung und subjektives Wohlbefinden in einer Längsschnittperspektive betrachtet. Die grundlegende Forschungsfrage lautet, welchen Veränderungen das subjektive Wohlbefinden im Zuge der Bildungsexpansion unterworfen war. Dabei werden entsprechend des A-P-K-Ansatzes (vgl. Mayer/Huinink 1990) sowohl Kohorten- und Alterseffekte als auch Periodeneffekte Berücksichtigung finden. Abhängige Variable ist die Lebenszufriedenheit als Repräsentation des globalen subjektiven Wohlbefindens; die Gesundheitszufriedenheit als wesentlicher Teilaspekt dient als Kontrollvariable.

Zusätzlich zu inhaltlichen Problemstellungen wird dem hier zugrunde gelegten methodischen Ansatz der Analyse von Variationen im Zeitverlauf besonderes Augenmerk geschenkt. Neben multivariaten Regressionsmodellen unter Berücksichtigung von Alters-, Perioden- und Kohorteneffekten (vgl. u. a. Bürklin/Klein/Ruß 1994; Hadjar/Becker 2006) hat sich in den letzten Jahrzehnten bei der Erforschung zeitlicher Veränderungen mit Panel-Daten die Mehrebenenanalyse als neues Datenanalyseverfahren in den Sozialwissenschaften etabliert (Bryk/Raudenbusch 2002; Klein/Pötschke 2004).

\section{Theoretischer Rahmen}

\subsection{Bildung und subjektives Wohlbefinden}

Das subjektive Wohlbefinden als übergeordneter Begriff bezieht sich darauf, ,how people evaluate their lives - both at the moment and for longer periods" (Diener/Oishi/Lucas 2003: 404). Es ist Ausdruck der Lebensqualität von Menschen und beschreibt, ob Menschen sich selbst als glücklich empfinden. ${ }^{1}$ Es spiegelt die Lebensbedingungen aus Sicht der jeweils Betroffenen wider (Spellerberg/Landua/Habich 1992: 251). Interessant für die Analyse längerfristiger Veränderungen ist das habituelle Wohlbefinden, das - im Gegensatz zum aktuellen Wohlbefinden - von einer vergleichsweise stärkeren zeitlichen

1 Schon gegen Ende der 1970er Jahre konnte die Forschung zum subjektiven Wohlbefinden Faktoren identifizieren, die mit dem Glückszustand des Menschen korrelieren. Glückliche Personen waren demnach u. a. jung, gesund und gebildet (vgl. Wilson 1967). 
Stabilität ist und das für eine Person typische Wohlbefinden über einen bestimmten Zeitraum darstellt (Diener/Larsen 1984). ${ }^{2}$ Es besteht aus einer kognitiven (Lebenszufriedenheit) und einer affektiven Komponente (Gefühlsbalance, Glücksempfinden); entsprechend gehören zu den meistgenutzten Indikatoren für die Quantifizierung des subjektiven Wohlbefindens Glück und Zufriedenheit (vgl. Noll 1989: 29 ff.). Allerdings existiert keine allgemein verbindliche Definition des Begriffes „Wohlbefinden“, und auch eine Abgrenzung zu verwandten Konzepten wie „Lebensqualität“ und „Glück“ erweist sich als schwierig (vgl. Veenhoven 2001).

Die Lebenszufriedenheit ist das kognitive Element des subjektiven Wohlbefindens und bezieht sich auf die individuelle Einschätzung der eigenen Lebenslage (vgl. Mayring 1991). Sie stellt eine Zusammenfassung der Zufriedenheiten in einzelnen Lebensbereichen dar - „eine Art Bilanz der Zufriedenheit mit der Familie, dem Beruf, dem Lebensstandard und den anderen Bereichszufriedenheiten“ (Glatzer/Zapf 1984a: 19), wobei von der jeweiligen Lebenssituation abhängt, welche Bereichszufriedenheiten besonders ausschlaggebend für die globale Einschätzung sind (Glatzer 1984: 237). Die Lebenszufriedenheit - als Verhältnis zwischen Anspruchsniveau und wahrgenommener Situation (Campbell 1981) - ist Ergebnis des Abwägens von Zuständen in Vergangenheit, Gegenwart und Zukunft und schließt soziale Vergleiche ein (Spellerberg/Landua/Habich 1992: 258). Zufriedenheit stellt sich ein, wenn Bedürfnisse subjektiv als befriedigt angesehen werden, also eine minimale Diskrepanz zwischen Anspruch und Wirklichkeit besteht. Sie ist daher in hohem Maße von individuellen Fähigkeiten der Wahrnehmung und der Informationsverarbeitung bestimmt (vgl. Schwarz/Strack 1985).

Bei den Bestimmungsfaktoren der Lebenszufriedenheit kommt der Gesundheit bzw. der Zufriedenheit mit der Gesundheit eine besondere Bedeutung zu (Glatzer/Zapf 1984a: 18). Die Gesundheitszufriedenheit - definiert als die Diskrepanz zwischen den (altersspezifischen) individuellen Ansprüchen an die eigene Gesundheit und der Wahrnehmung des eigenen Gesundheitszustands bzw. der Körperfunktionen - wirkt sich ,auf die allgemeine Lebenszufriedenheit“" aus (Dehlinger/Ortmann 1992: 93). In der vorliegenden Analyse wird der Aspekt der Gesundheitszufriedenheit als eine unabhängige Variable in die Modelle zur Erklärung der Lebenszufriedenheit eingeführt, um herauszuarbeiten, welche Rolle der Zufriedenheit mit der Gesundheit innerhalb des subjektiven Wohlbefindens zukommt, und um die verschiedenen zeitlichen Veränderungsprozesse unter Kontrolle des Gesundheitsaspekts - das heißt losgelöst von zwar sozial beeinflussten, aber letztlich biologischen Alterungsprozessen - betrachten zu können.

Einen theoretischen Zugang zu den Bestimmungsfaktoren subjektiven Wohlbefindens bietet die Theorie der sozialen Produktionsfunktionen in der Explikation von Lindenberg und Frey (1993) und Ormel et al. (1999). Wohlbefinden sozialer und physischer Natur ist nach dieser Theorie das zentrale Ziel menschlichen Handelns und kann durch die Erfüllung bestimmter instrumenteller Ziele (Stimulation, Komfort, Status, Verhaltensbestätigung und Affekt) produziert werden. Inwieweit diese Zwischenziele erreicht

2 Der Begriff des habituellen Wohlbefindens ist dennoch nicht so zu verstehen, dass das Wohlbefinden weitgehend unabhängig von den Lebensbedingungen wäre. Die Perspektive wird durch den Begriff des „Habituellen“ nur von einer flüchtigen Momentaufnahme zu einer stärker reflektierten Globaleinschätzung hingelenkt. 
Abbildung 1: Wohlbefinden und soziale Produktionsfunktionen

\begin{tabular}{|c|c|c|c|c|c|}
\hline \multicolumn{6}{|c|}{ Subjektves Wohlbefinden } \\
\hline Universelle & \multicolumn{2}{|c|}{ Physisches Wohlbefinden } & \multicolumn{3}{|c|}{ Soziales Wohlbefinden/Soziale Anerkennung } \\
\hline $\begin{array}{l}\text { Ziele erster } \\
\text { Ordnung }\end{array}$ & $\begin{array}{c}\text { Stimulation/ } \\
\text { Aktivation } \\
\text { (optimales } \\
\text { Erregungsniveau) }\end{array}$ & $\begin{array}{c}\text { Komfort } \\
\text { (Absenz } \\
\text { physiologischer } \\
\text { Bedürfnisse, } \\
\text { angenehmes und } \\
\text { sicheres Umfeld) }\end{array}$ & $\begin{array}{l}\text { Status (Kontrolle } \\
\text { über knappe } \\
\text { Ressourcen) }\end{array}$ & $\begin{array}{l}\text { Verhaltens- } \\
\text { bestätigung } \\
\text { (Anerkennung, } \\
\text { die richtigen } \\
\text { Dinge zu tun) }\end{array}$ & $\begin{array}{c}\text { Affekt } \\
\text { (positive } \\
\text { Zuwendung durch } \\
\text { Andere) }\end{array}$ \\
\hline $\begin{array}{l}\text { Produktions- } \\
\text { mittel }\end{array}$ & $\begin{array}{l}\text { physische und } \\
\text { mentale } \\
\text { Aktivitäten zur } \\
\text { Produktion von } \\
\text { Erregung }\end{array}$ & $\begin{array}{c}\text { Absenz von } \\
\text { Schmerz/Durst/ } \\
\text { Hunger/ } \\
\text { Erschöpfung; } \\
\text { Vitalität, gute } \\
\text { Wohnverhältnisse, } \\
\text { soziale Wohlfahrt, } \\
\text { Sicherheit }\end{array}$ & $\begin{array}{l}\text { Beruf, Lebensstil, } \\
\text { Exzellenz in Sport } \\
\text { oder Arbeit }\end{array}$ & $\begin{array}{l}\text { Konformität mit } \\
\text { externalen und } \\
\text { internalen } \\
\text { Normen }\end{array}$ & $\begin{array}{c}\text { intime } \\
\text { Beziehungen, } \\
\text { emotionale } \\
\text { Untertützung }\end{array}$ \\
\hline Ressourcen & $\begin{array}{l}\text { physische und } \\
\text { mentale } \\
\text { Anstrengungen }\end{array}$ & $\begin{array}{l}\text { Nahrung, } \\
\text { Gesundheits- } \\
\text { fürsorge, Geld }\end{array}$ & $\begin{array}{l}\text { Bildung, soziale } \\
\text { Schicht, } \\
\text { besondere } \\
\text { Fähigkeiten }\end{array}$ & $\begin{array}{l}\text { soziale } \\
\text { Fähigkeiten, } \\
\text { Kompetenz }\end{array}$ & $\begin{array}{c}\text { Partner, Empathie, } \\
\text { Attraktivität }\end{array}$ \\
\hline
\end{tabular}

Quelle: Ormel et al. (1999: 67)

werden, hängt von individuell verfügbaren Ressourcen als Mittel zur Produktion von Wohlbefinden sowie von Verhaltenslimitierungen (,,constraints“) ab. In Abbildung 1 sind die jeweiligen Ziele und die Ressourcen, die zur Zielerreichung beitragen, dargestellt.

Wenngleich innerhalb der Theorie der sozialen Produktionsfunktionen Bildung und Fähigkeiten nur in Bezug auf die Zwischenziele Status und Verhaltensaffekt thematisiert werden, ist davon auszugehen, dass Bildung als Ressource fungiert, welche die Produktion aller Zwischengüter unterstützt. Zur theoretischen Plausibilisierung der Bedeutung von Bildung als Ressource zur Produktion subjektiven Wohlbefindens eröffnen sich - in Anlehnung an Becker (1998) oder Hadjar (2006) - zwei Zugangswege: Zum einen ist Bildung als über Sozialisationsprozesse weitergegebenes Humanvermögen zu fassen (vgl. ebd.: 206 ff.). In dieser Sichtweise bedeutet eine höhere Bildung erweiterte kognitive Fähigkeiten und Handlungskompetenzen, was mit einer effizienteren Aneignung entwicklungsrelevanter Wissensbestände und damit besseren Voraussetzungen für die Lebensgestaltung und die Partizipation in verschiedenen gesellschaftlichen Bereichen einhergeht (Mayer 1994). Ein höheres Bildungsniveau ist also mit einem besseren Wissen verknüpft, wie und über welche Ressourcen die Zwischengüter Stimulation, Komfort, Status, Verhaltensbestätigung und Affekt produziert werden können. Mit einer höheren Bildung sind aus Sicht der Humanvermögen-These sowohl eine bessere Befähigung zur Selbstdisziplinierung und zur rationalen Lebensführung verbunden als auch bessere Fähigkeiten zur „Antizipation und Verarbeitung kritischer Lebensereignisse sowie Bewältigung vorhandener Stressoren, die unter anderem Lebenszufriedenheit, Selbstwertgefühl und Gesundheit beeinträchtigen“ (Becker 1998: 138). Zum anderen erscheint Bildung aus einer ökonomischen Perspektive als $\mathrm{Hu}$ mankapital (Becker 1974): Bildung bzw. akkumulierte Bildungszertifikate können im spä- 
teren Erwerbsleben in Positionen auf dem Arbeitsmarkt umgesetzt werden, die bestimmte soziale Lagen nach sich ziehen. Über das erworbene Einkommen werden somit indirekt durch die Bildung sozioökonomische Lebenschancen bestimmt (vgl. Becker 1998). Ein höherer Bildungsstand geht - nach dem Statuszuweisungsansatz (Mayer/Blossfeld 1990) - mit einem höheren Status und entsprechend höheren Einkommen einher, was wiederum einen besseren Lebensstandard in Bezug auf Wohnverhältnisse, Freizeitaktivitäten, soziale Beziehungen, Hygiene, Ernährung, Gesundheitsvorsorge und -versorgung bzw. gesundheitsfördernde Lebensstile nach sich zieht (Abel 1999). Hier stellt sich Bildung als Grundlage für materielle und statusbezogene Ressourcen dar, die der Erzielung der Zwischenziele im Sinne der Theorie der sozialen Produktionsfunktionen (Ormel et al. 1999) dienen.

Empirische Befunde stützen sowohl die Humanvermögen-These als auch die Humankapital-These: Höher gebildete Individuen sind zufriedener mit dem Leben bzw. weisen ein höheres subjektives Wohlbefinden auf (Bradburn 1969; Oswald 1997). In gleicher Weise gilt das aber auch für Personen mit einem höheren Status und einem höheren Einkommen (Spellerberg/Landua/Habich 1992: 258). ${ }^{3}$ Zusammenfassend ist anzunehmen, dass Individuen mit höherer Bildung - einerseits infolge besserer kognitiver Fähigkeiten (Humanvermögen-These), andererseits aufgrund einer statusbedingten besseren Ausstattung mit ökonomischen Ressourcen (Humankapital-These) - höhere Chancen haben, ihre Bedürfnisse zu befriedigen und die instrumentellen Zwischenziele zur Produktion von subjektivem Wohlbefinden zu bedienen. ${ }^{4}$

Hypothese 1: Je höher der Bildungsstand eines Individuums ist, desto höher ist seine Lebenszufriedenheit.

\subsection{Subjektives Wohlbefinden im Zeitverlauf: Kohorten-, Alters- und Periodeneinflüsse}

Für die Analyse der Entwicklung des subjektiven Wohlbefindens ist eine Längsschnittperspektive unerlässlich. Dabei ist die Berücksichtigung dreier temporaler Aspekte sinnvoll, denn Entwicklungen können sowohl über die Kohortenabfolge (Kohorteneffekte) als auch über die Erhebungszeitpunkte (Periodeneffekte) sowie den Lebenszyklus (Alterseffekte) erfolgen. Soziale Tatbestände werden nach dem A-P-K-Ansatz nicht als geschichtslose Zustände begriffen, sondern als Komposition der Effekte des Alterns, der Sozialisation und der strukturellen Einflüsse zum Zeitpunkt der Untersuchung.

3 In Vorgriff auf die Kontrolle der Gesundheitszufriedenheit in den Modellen zur Analyse der Lebenszufriedenheit ist auf empirische Evidenzen zu bildungsspezifischen Unterschieden im Gesundheitszustand und klassen- und statusspezifischen Krankheitsrisiken hinzuweisen. Höher Gebildete sind weniger depressiv, gehen besser mit kritischen Lebensereignissen um, vermeiden Gesundheitsrisiken und leben letztlich länger (vgl. Becker 1998: 122f.; Mueller/ Heinzel-Gutenbrunner 2001: 28f.; Klein/Unger/Schulze 2006).

4 Im Gegensatz dazu könnte angenommen werden, dass ein höheres Bildungsniveau die Kenntnisse von Alternativen erweitert und die Ansprüche erhöht, was zu einer relativen Deprivation führt. Mit der anhaltenden Bildungsexpansion würde also das Zufriedenheitsniveau verringert werden. Diese Annahmen konnten jedoch empirisch nicht plausibilisiert werden (Glatzer/Zapf 1984a: 18). 
Kohorteneffekte auf das subjektive Wohlbefinden. Kohorteneffekte bzw. Sozialisationseffekte bezeichnen Unterschiede, die sich auf die Zugehörigkeit zu verschiedenen Generationen zurückführen lassen. Bestimmte individuelle Merkmale (z. B. Werte, Bildung) werden aus dieser Perspektive vor allem im Kindes- und Jugendalter, das heißt in der formativen Phase des Sozialisationsprozesses erworben, unterliegen in der weiteren Persönlichkeitsentwicklung nur wenigen Veränderungen und sind damit über die gesamte Lebensspanne wirksam (vgl. Ryder 1965: 856; Inglehart 1997).

Die kohortenspezifische Betrachtung des subjektiven Wohlbefindens setzt somit bei erworbenen Merkmalen an, die mehr oder weniger alle Mitglieder einer bestimmten Kohorte kennzeichnen. Im Hinblick auf das subjektive Wohlbefinden wird die Herausbildung bestimmter Mentalitäten im Zuge der Sozialisation angenommen (vgl. Geiger 1987: 77). So lässt sich die Argumentation von Veenhoven (1995: 34) bezüglich kultureller Einflüsse auf Kohorteneffekte umdeuten: „If hardship (...) has brought on a pessimistic outlook on life (...), that outlook may persist for quite some time, and will discourage a positive judgement of life." Der hinter diesem Befund stehende soziale Mechanismus lässt sich mit der Theorie der kognitiven Dissonanz (Festinger 1957) fassen: Prägende Lebensereignisse in der Sozialisation und das damit verbundene Niveau an subjektivem Wohlbefinden beeinflussen die Wahrnehmung und Bewertung späterer Situationen. So sollte das Aufwachsen in relativem Wohlstand, wie es die ab den 1950ern geborenen Deutschen erleben konnten, ein höheres Grundniveau an subjektivem Wohlbefinden nach sich ziehen. Um dieses Niveau „kreist“ im weiteren Lebensverlauf das Wohlbefinden (Baier/Boehnke 2007).

Im Hinblick auf den fokussierten Bildungseffekt sind die Kohorten vor dem Hintergrund der Bildungsexpansion und der kohortenspezifischen Quote der Höhergebildeten zu deuten. ${ }^{5}$ Im Kern der Argumentation steht dabei der Prozess der ,kognitiven Mobilisierung“" (Baumert 1991: 347; Baumert et al. 2003) als eine zentrale (erwünschte) Folge der Bildungsexpansion, der durch eine Zunahme an individuellen Fähigkeiten der Informationsverarbeitung und an Handlungskompetenzen gekennzeichnet ist. Mit der Bildungsexpansion hat eine kognitive Mobilisierung dergestalt stattgefunden, dass über die Kohortenabfolge die Kompetenzen, „seines eigenen Glückes Schmied zu sein“ bzw. eine Bedürfnisbefriedigung zu erreichen, und damit das subjektive Wohlbefinden zugenommen haben (Müller 1998).

Als hemmender Faktor dieser Entwicklung kristallisiert sich eine unerwünschte Folge der Bildungsexpansion heraus: So befürchtete bereits Schelsky (1956), dass bei einer sozialen Öffnung der höheren Schullaufbahnen und der Heterogenisierung der Schülerschaft an höheren Schulen das Risiko sinkender Standards in Kauf genommen werden müsse. Die Gruppe der höher Gebildeten nimmt hinsichtlich ihrer Fähigkeiten, Kompetenzen, Orientierungen und Verhaltensmuster eine weniger distinkte Position gegenüber den geringer Gebildeten ein (Baumert 1991). Dies führt zwar nicht zur Negierung des Prozesses der kognitiven Mobilisierung, aber zu dessen Abschwächung. Empirisch sollte sich die Tendenz sinkender Standards in höheren Schulen in einer leichten Annäherung des subjektiven Wohlbefindens in den niedrigen und höheren Bildungsschichten

5 Im Zuge der Bildungsexpansion ist seit Mitte der 1960er Jahre eine Zunahme qualifizierter Berufsabschlüsse, der Abiturientenquote und der Studierendenzahlen zu verzeichnen (vgl. Baumert 1991; Müller 1998). 
ausdrücken. Ausgeschlossen von dieser Annäherung sind jedoch die besonders gering Gebildeten als Verlierer der Bildungsexpansion; deren negative Distinktion sollte in Anbetracht der Homogenisierung der Schülerschaft von Hauptschulen als „Restschule“ (Solga/Wagner 2001) erhalten bleiben bzw. sogar zunehmen.

Aus der bildungsspezifischen Kohortendeutung ergibt sich eine ähnliche These wie aus der Interpretation der Kohorten nach den Bedingungen des Aufwachsens: Die älteren Kohorten mit einem tendenziell niedrigeren Bildungsstand sind gegenüber den jüngeren, höher gebildeten Kohorten hinsichtlich der Kompetenzen zur Lebensgestaltung und Zielerreichung benachteiligt. Erste Anzeichen einer kognitiven Mobilisierung sind für die Wirtschaftswundergeneration der in den 1940ern Geborenen zu erwarten, die bereits vom Ausbau des mittleren Schulwesens profitierte. Die eigentlichen Generationen der Bildungsexpansion sind die Geburtsjahrgänge der 1950er und 1960er Jahre, denn hier stieg die Abiturientenquote rasant an. Auf den ersten Blick sollten bei diesem hypothetischen Szenario die Kohorteneffekte ganz in den Bildungseffekten aufgehen und entsprechend nicht signifikant sein. Es ist aber zusätzlich davon auszugehen, dass die Bildungsexpansion über Aggregatmechanismen (Massenmedien, technischer Fortschritt etc.) den gesamten Kohorten Vorteile gebracht hat. Mit Blick auf den Zusammenhang zwischen kognitiver Mobilisierung und subjektivem Wohlbefinden ist also anzunehmen:

Hypothese 2: Über die Kohortenabfolge steigt die Lebenszufriedenheit an.

Die Überlegungen zur Heterogenisierung der höheren Bildungsschichten bzw. der Homogenisierung der niedrigen Bildungsschichten werden im Rahmen der folgenden Hypothese gefasst:

Hypothese 3: Über die Kohortenabfolge nähern sich die höheren Bildungsschichten in ihrer Lebenszufriedenheit den niedrigeren Bildungsschichten tendenziell an, wobei die Distinktion der Individuen ohne Ausbildung erhalten bleibt.

Alterseffekte auf das subjektive Wohlbefinden. Alterseffekte beschreiben Wandlungsprozesse - hier Veränderungen im subjektiven Wohlbefinden - aufgrund der Stellung eines Menschen im Lebensverlauf und werden daher auch Lebenszykluseffekte genannt (Klecka 1971; Bürklin/Klein/Ruß 1994). Aus soziologischer Perspektive stehen hinter ihnen altersspezifische soziale Erfahrungen innerhalb eines lebenslangen Sozialisationsprozesses oder Prozesse der sozialstrukturellen Etablierung im Lebensverlauf, vor allem im Hinblick auf das Erwerbs- und Familienleben.

Der Betrachtung von Einflüssen des Alters auf das subjektive Wohlbefinden kann keine eindeutige Annahme über einen Zusammenhang zugrunde gelegt werden. Empirische Befunde (vgl. u. a. Bradburn 1969; Grob 1995) legen nahe, dass sich das globale subjektive Wohlbefinden über die Lebensspanne nur unbedeutend ändert: Junge Menschen haben stärkere Glücksgefühle; ältere Menschen sind zufriedener, da mit zunehmendem Lebensalter viele Ziele im Leben bereits erreicht wurden (vgl. Argyle 1987). In dieser Argumentation würde der Zusammenhang zwischen subjektivem Wohlbefinden und Alter einem eher u-förmigen Verlauf entsprechen (vgl. Oswald 1997). ${ }^{6}$ Aufgrund zweier gegenläufiger Tendenzen kann kein eindeutiger Alterseffekt angenommen wer-

6 Dieser u-förmige Verlauf des subjektiven Wohlbefindens - hohes Wohlbefinden im Jugendalter, Tiefpunkt um das 40. Lebensjahr und monotones Ansteigen danach - wird von Blanch- 
den: Zum einen lassen die zunehmende Integration in Arbeitsmarkt und Familienleben, ein höheres Einkommen und eine hohe Selbstwirksamkeit die Diskrepanz zwischen angestrebten und erreichten Zielen sinken und so die globale Lebenszufriedenheit ansteigen (Andrews/Withey 1976; Veenhoven 1997). Zum anderen ist jedoch mit einem hohen Alter - infolge schlechterer Gesundheit, der Abnahme sozialer Unterstützung und dem Alleinleben - das Gleichgewicht zwischen Anspruch und Wirklichkeit nicht mehr zu halten, was die Lebenszufriedenheit absinken lässt (Grob 1995). Ursächlich für die oft zitierte höhere Lebenszufriedenheit älterer Menschen könnte sein, dass sie im Laufe ihres Lebens Ansprüche und Erwartungen einander angepasst haben. Empirisch konnte das nicht bestätigt werden (Glatzer/Zapf 1984a: 18). Gerade im höheren Alter spielt die abnehmende Zufriedenheit mit der Gesundheit eine tragende Rolle. So zeigen Analysen von Mohr (1987: 178), dass „,bei den alten Menschen vor allem die wahrgenommene gesundheitliche Beeinträchtigung" die Lebenszufriedenheit determiniert. Lang und Müller-Andritzky (1984: 146) zeigen zudem, dass Kränkere von einer niedrigeren subjektiven Lebenszufriedenheit berichteten. ${ }^{7}$

Da der Aspekt der Gesundheitszufriedenheit in den Modellen kontrolliert, das heißt als eigenständige unabhängige Variable eingeführt wird, bezieht sich der hier in eine Hypothese zu fassende Alterseffekt nur noch in geringem Maß auf die Entwicklung der Gesundheit im Lebenszyklus. Angesichts der verschiedenen gegenläufigen Befunde und weil der Gesundheitsaspekt getrennt betrachtet wird, ergibt sich die These, dass - bei Betrachtung des gesamten Lebenszyklus - das Alter keinen statistisch signifikanten Einfluss auf die Lebenszufriedenheit hat. ${ }^{8}$

Hypothese 4: Das Alter hat keinen Einfluss auf die Lebenszufriedenheit.

Periodeneffekte auf das subjektive Wohlbefinden. Periodeneffekte sind schließlich Einflüsse des historischen und gesellschaftlichen Kontextes. Sie erklären sich aus sozialen Umweltfaktoren, wie Einflüssen des Werteklimas einer Gesellschaft bzw. bestimmter sozialstruktureller Veränderungen, die auf Individuen und ihre Merkmale und Alterungsprozesse wirken (Wagner 2001: 12). Sie sind somit wie die Kohorteneffekte mit gesellschaftlichen Ereignissen und Zuständen verknüpft, die sich allerdings auf den Messzeitpunkt der Daten beziehen.

flower und Oswald (2004) mit einer Anpassung des Anspruchsniveaus nach der Lebensmitte erklärt.

7 Der in bivariaten Korrelationsanalysen zumeist aufscheinende negative Zusammenhang zwischen Alter und Gesundheitszufriedenheit ist nicht eindeutig belegt (vgl. Schneider 1999). So konstatieren Ferraro (1980) oder Borchelt et al. (1996) bei Berücksichtigung von Drittvariablen einen positiven Zusammenhang zwischen Alter und Gesundheitszufriedenheit: Ältere Menschen sind offenbar nicht per se unzufriedener mit ihrer Gesundheit, sondern bei Kontrolle der Anzahl der Erkrankungen und ihres Bildungsstandes sogar tendenziell zufriedener als jüngere. Andererseits weisen die Befunde von Schneider (1999) auf einen negativen Zusammenhang zwischen Alter und Gesundheitszufriedenheit hin, der auch bei einer Drittvariablenkontrolle erhalten bleibt.

8 Auch bisherige Analysen zur Veränderung der Lebenszufriedenheit auf der Datenbasis des Sozio-Ökonomischen Panels weisen auf eine erstaunliche Stabilität der individuellen Skalenwerte im Zeitverlauf hin (Noll 1989: 33). 
Die Periode bzw. der Erhebungszeitpunkt wird in die folgenden Analysen zum Wandel des subjektiven Wohlbefindens nicht als zeitliche Variable integriert, da ein simultaner Test von zeitlichen Alters-, Kohorten- und Periodeneffekten infolge der Konfundierung der drei Variablen nicht möglich ist. Dieses Problem wird umgangen, indem die Periodenvariable durch eine theoretisch sinnvolle Makro-Variable ersetzt wird (vgl. Glenn 2005; Hadjar/Becker 2006). Das subjektive Wohlbefinden ist von der individuellen Wahrnehmung der periodenspezifischen Chancen zur Befriedigung der Bedürfnisse abhängig. Eine bessere gesellschaftliche Lage bietet höhere Chancen zur Bedürfnisbefriedigung und damit bessere Entfaltungsmöglichkeiten und ermöglicht so eine höhere „Lebbarkeit“ (Veenhoven 1997: 268) bzw. ein höheres subjektives Wohlbefinden auf der individuellen Ebene. Eine schlechte gesellschaftliche Lage ist demgegenüber mit Unzufriedenheit mit dem Leben und psycho-somatischen Beschwerden (Stichwort: Gesundheitszufriedenheit) verbunden. Ein besonderes Gewicht bei der Bestimmung der gesellschaftlichen Lage kommen der ökonomischen Leistungsfähigkeit und der Arbeitslosigkeit zu. Die für das subjektive Wohlbefinden relevanten Bedingungen sind in reichen Gesellschaften besser als in armen; die ökonomische Leistungsfähigkeit eines Landes korreliert positiv mit dem subjektiven Wohlbefinden (Inglehart/Klingemann 2000; Veenhoven 2001).

Im Rahmen der folgenden Analysen wird die spezifische Arbeitslosenquote in Westdeutschland als Periodenmerkmal spezifiziert. Zum einen wird die Arbeitslosenquote als Ausdruck des gesellschaftlichen Zustands - im Gegensatz zu anderen wirtschaftlichen Kennzahlen wie z.B. Wachstumsraten - in der Bevölkerung breit perzipiert, was eine Relevanz dieses Indikators für die Ausprägung des subjektiven Wohlbefindens plausibel erscheinen lässt. Die Arbeitslosenzahlen stehen für die ,wichtigsten Auswirkungen, welche die Veränderungen der Konjunkturlage auf die objektive persönliche Betroffenheit“ und letztlich auf die subjektiven Wahrnehmungen vieler Bundesbürger haben (Terwey 1990: 145). Zum anderen beeinflusst Arbeitslosigkeit auf der individuellen Ebene das subjektive Wohlbefinden, was im Sinne der Aggregationslogik (Büschges/Abraham/Funk 1996: 101) eine Wirkung auf die gesellschaftliche Ebene hat. Arbeitslosigkeit stellt sich in empirischen Studien (Oswald 1997; Di Tella/MacCulloch/Oswald 2001) häufig als der im Vergleich zu anderen Einflussfaktoren (z.B. Familienstand, Einkommen) stärkste Prädiktor eines sinkenden subjektiven Wohlbefindens heraus. Von Arbeitslosigkeit Betroffene weisen eine geringere Lebenszufriedenheit auf (Clark/Oswald 1994; Winkelmann/Winkelmann 1998). Das Zufriedenheitsniveau liegt sogar unterhalb von dem anderer Problemgruppen wie Alleinerziehenden, Schwerbehinderten oder Alleinlebenden, wobei die Lebenszufriedenheit in doppelter Hinsicht durch die Erwerbskonstellation beeinflusst wird: „,zum einen indirekt, über die Verringerung des Haushaltseinkommens, zum anderen direkt als Ausdruck des sozialen Ausschlusses aus dem Erwerbsleben“ (Krause 1987: 120). Arbeitslosigkeitserfahrungen reduzieren das subjektive Wohlbefinden auf Dauer, wobei sich mehrmalige Job-Verluste besonders negativ auswirken. Empirisch zeigen sich keine Anpassungs- bzw. Gewöhnungseffekte - etwa dergestalt, dass zur Kompensation der Arbeitslosigkeitserfahrung das Anspruchsniveau bezüglich des subjektiven Wohlbefindens sinken würde (Clark/Oswald 1994). ${ }^{9}$ Die negative Wirkung der Arbeitslosigkeit übertrifft

9 Anpassungseffekte des Anspruchsniveaus sind jedoch schwer nachzuweisen, weil Informationen über die Wahl, die Wichtigkeit und die Merkmale der Referenzgruppen fehlen. 
sogar den Einfluss schlecht bezahlter Arbeit auf das subjektive Wohlbefinden, da offenbar Arbeitslosigkeit einen sehr geringen ökonomischen und im Hinblick auf die soziale Identität auch sehr geringen psychischen Nutzen hat (Frey/Stutzer 2002). ${ }^{10}$

Aus diesen Makro- und Mikroannahmen ergibt sich die Hypothese, dass das individuelle subjektive Wohlbefinden von der aggregierten Arbeitslosigkeit (Arbeitslosenquote) negativ beeinflusst wird.

Hypothese 5: Je höher die Arbeitslosenquote zu einem Erhebungszeitpunkt ist, desto geringer ist die Lebenszufriedenheit.

Um ökologische Fehlschlüsse - das heißt die fälschliche Übertragung von Befunden auf der Makroebene auf die Mikroebene - zu vermeiden, wird die Arbeitslosenquote auf der Makroebene in den multivariaten Modellen kontrolliert durch die individuelle Arbeitslosigkeit. Nur so kann gezeigt werden, ob Arbeitslosigkeit auch auf der individuellen Ebene die sich auf der Makroebene abzeichnenden Wirkungen hat und die Effekte individueller und allgemeiner Arbeitslosigkeit (ebd.) gleich gerichtet sind. Während die Arbeitslosenquote als Periodenmerkmal die allgemeine Stimmung in der Bevölkerung beeinflusst, ist die individuelle Arbeitslosigkeit ein Faktor, der direkt auf das individuelle subjektive Wohlbefinden wirkt. Die Arbeitslosenquote und die individuelle Arbeitslosigkeit sind insofern entkoppelt, als sie sich auf verschiedene Ebenen beziehen. ${ }^{11}$

Wie bereits erläutert, wird zudem in den einzelnen Modellen zur Erklärung des subjektiven Wohlbefindens die Gesundheitszufriedenheit kontrolliert, die eine wichtige Rolle spielt. Daher soll dieser Aspekt von den anderen Effekten abgetrennt werden. Im Hinblick auf die Richtung des eigenständigen Effekts der Gesundheitszufriedenheit auf die Lebenszufriedenheit ist von einer positiven Wirkung auszugehen. So zeigen u.a. Dehlinger und Ortmann (1992), dass eine positive Wahrnehmung der Gesundheit mit einem höheren subjektiven Wohlbefinden verbunden ist. Die Bedeutung der Gesundheitszufriedenheit für das globale subjektive Wohlbefinden nimmt über den Lebensverlauf hin zu. Im höheren Alter wird die Lebenszufriedenheit sehr stark durch die dann rapide abnehmende Gesundheit beeinträchtigt (vgl. Schneider 1999).

\section{A-P-K-Mehrebenenanalyse zur Untersuchung von Längsschnittfragestellungen}

Die Analysen zur Lebenszufriedenheit werden anhand von Mehrebenenmodellen durchgeführt unter Heranziehung des A-P-K-Ansatzes (Mayer/Huinink 1990), der eine Synthese aus Längs- und Querschnittsanalyse darstellt und u.a. der Bestimmung des relativen Einflusses von Alters-, Perioden- und Kohorteneffekten auf Veränderungen von Einstellungen und Verhaltensweisen dient (vgl. Hadjar/Becker 2006).

10 Zudem wirkt Arbeitslosigkeit als Stressor, der Gesundheitsprobleme auslösen kann. So stellen Mueller und Heinzel-Gutenbrunner (2001: $30 \mathrm{ff}$.) fest, dass vor allem Langzeitarbeitslose im Hinblick auf psycho-somatische Beschwerden einen schlechteren Gesundheitszustand haben.

11 Zum besseren Verständnis lässt sich hier auch das Bedrohungskonzept (,,integrated threat theory“; Stephan/Stephan 2000) heranziehen. Darin werden nationale Bedrohungen - z.B. steigende Preise, höhere Arbeitslosenquoten - von individuellen Bedrohungen - z.B. durch individuelle Arbeitslosigkeit oder Krankheit - unterschieden (vgl. Rippl et al. 2005). 


\subsection{A-P-K-Analyse}

Ziel der A-P-K-Analyse ist es, Veränderungen in zeitlich sukzessiv erhobenen Merkmalsausprägungen anhand von $\mathrm{zu}$ ähnlichen Zeitpunkten geborenen Personengruppen über ihren Lebensverlauf hinweg nachzuvollziehen (Plum 1982: 510; Wagner 2001), wobei ökologische bzw. temporale Fehlschlüsse durch die simultane Betrachtung der drei temporalen Einflussgrößen vermieden werden. ${ }^{12}$

Ein Spezifikum dieses Verfahrens liegt in der Dekomposition der zeitlichen Effekte. Werden bei Querschnittsuntersuchungen Unterschiede beispielsweise zwischen Altersgruppen aufgezeigt, so könnten diese zum Teil oder vollständig auf die Zugehörigkeit zu verschiedenen Kohorten zurückgehen. Daher führt nur eine simultane (Längsschnitt-) Analyse von Alters-, Perioden- und Kohorteneffekten zu einer vollständigen Trennung der verschiedenen Einflüsse. Das lineare Verhältnis, in dem die drei zeitlichen Komponenten stehen (Periode $=$ Kohorte + Alter), führt bei Aufnahme aller drei Effekte in ein Modell zu einem Identifikationsproblem (Glenn 2005: 6; Mayer/Huinink 1990). Um die Konfundierung von Alters-, Perioden- und Kohorteneffekten und damit auch die perfekte Multikollinearität im Regressionsmodell aufzulösen, können zwei Wege beschritten werden: Zum einen kann theoretisch begründet argumentiert werden, dass mindestens einer der drei Faktoren keinen originären Einfluss hat und daher von der Analyse ausgeschlossen werden kann. Dies ist eine nicht unproblematische Einschränkung in der Untersuchung zeitlichen Wandels. Zum anderen „one can (...) include measures of the causal variables for which age, period and cohort are surrogates“ (Tuma/Hannan 1984: 192). Es werden also Variablen benötigt, die theoretisch begründbar das messen, was durch die Zeitvariablen gemessen werden soll, und diese dann ersetzen (Glenn 2005: 23; Wagner 2001). Da im Folgenden das subjektive Wohlbefinden im Zeitverlauf ganzheitlich analysiert werden soll, wird hier die zweite Strategie verfolgt.

\subsection{Analysen mit Mehrebenenmodellen}

Die Fragestellung zum subjektiven Wohlbefinden aus zeitlicher Perspektive soll unter Heranziehung des Paneldatensatzes (SOEP), einer Haushaltsstichprobe, beantwortet werden. Da die wiederholten Messungen an den gleichen Personen (Individueneffekt) sowie die einzelnen in einem Haushalt lebenden Personen nicht voneinander unabhängig sind (Stichwort: Autokorrelation), erscheint die Mehrebenenanalyse als adäquates Analyseverfahren.

Theoretische Grundlagen. Die Verbindung von individueller und gesellschaftlicher Ebene bzw. von Mikro- und Makrosichtweise ist ein wesentliches Anliegen klassischer und zeitgenössischer sozialwissenschaftlicher Ansätze und erweist sich bezüglich vieler Fragestellungen als nahezu unerlässlich (vgl. Coleman 1991; Hedström/Swedberg 1998).

12 Unter einem temporalen Fehlschluss soll die fälschliche Annahme eines zeitlichen Effekts, der sich in der A-P-K-Modellierung als Effekt anderer temporaler Dimensionen herausstellt, verstanden werden. So können etwa Kohorten- oder Periodeneffekte auf Effekten des Alters (Lebenszyklus) bzw. der Altersverteilung basieren. 
Doch die simultane Analyse beider Ebenen ist im Hinblick auf das AggregationsDisaggregationsdilemma (de Leeuw 2002) nicht unproblematisch: Die gängige Praxis, Individualdaten zu aggregieren, ist insofern problematisch, als dadurch viele Informationen verloren gehen. Nach der Aggregierung sind Aussagen nur noch auf der Gruppenund nicht mehr auf der Individualebene möglich, was zu ökologischen Fehlschlüssen im Sinne von Robinson (1950) führen kann (vgl. auch Snijders/Bosker 1999: 13 ff.). Ein ökologischer Fehlschluss liegt vor, wenn von Kollektiveigenschaften auf entsprechende Individualeigenschaften unmittelbar, also ohne die Kontexthypothesen zu überprüfen, geschlossen wird. Dieser Fehlschluss ist vermeidbar, wenn der Einfluss des Kontextes auf individuelles Verhalten untersucht wird, wie es in Mehrebenenanalysen geschieht.

Die Notwendigkeit einer adäquaten Mehrebenenmodellierung ergibt sich bereits aus dem wissenschaftstheoretischen Paradigma des methodologischen Individualismus, dass die Gesellschaft auf den Handlungen einzelner Individuen basiert. Der Mehrebenenansatz fügt hinzu, dass Menschen auch gesellschaftliche Objekte sind (Blien/Wiedenbeck 2002: 311). Mehrebenenanalysen sind in diesem Sinne nicht nur ein spezielles statistisches Modell, sondern setzen eine besondere theoretische Erklärung voraus (vgl. Markovsky 1987).

Statistische Grundlagen. Für die vorliegende Datenstruktur wie auch die Fragestellungen erscheint das statistische Verfahren der Mehrebenenanalyse adäquat. Es wurde entwickelt, um hierarchisch strukturierte Daten angemessen auszuwerten (vgl. Bryk/ Raudenbush 2002; Engel 1998; Kreft/de Leeuw 1998; Snijders/Bosker 1999). Dabei wird das Allgemeine Lineare Modell auf mehr als eine Analyseebene ausgeweitet; hierbei können mindestens zwei Ebenen, maximal aber auch drei oder mehr Ebenen simultan verrechnet werden. Die hierarchisch niedrigste Ebene ist die individuelle Einheit; zweite oder höhere Ebenen stellen kollektive Einheiten dar. Charakteristisch ist, dass die Einheiten der niedrigsten Ebene jeweils einer übergeordneten Ebene angehören und in diese eingebettet sind. Mit Hilfe der Mehrebenenmodellierung können Aussagen über die Entwicklung der Lebenszufriedenheit von Personen unter Berücksichtigung kontextueller Faktoren gemacht werden. Dies ist bedeutsam, da in Mehrebenenanalysen davon ausgegangen wird, dass sich die Personen innerhalb eines Kontextes in Bezug auf das Kriterium ähnlicher sind, als die Personen zwischen den Einheiten. Die Berücksichtigung der Beziehungen innerhalb der einzelnen Aggregateinheiten sowie der Beziehungen zwischen den Aggregateinheiten kommt u.a. darin zum Ausdruck, dass unterschiedliche Koeffizienten nicht als störend betrachtet werden, sondern Ausdruck unterschiedlicher Bedingungen und Prozesse sind (vgl. Kreft/de Leeuw 1998: 39 ff.). Die Varianz der Koeffizienten wird nun wiederum durch die Fehlerterme zu erklären versucht. Im Vergleich zum Allgemeinen Linearen Modell resultiert daraus eine erweiterte Fehlerstruktur: nämlich individuelle Fehler und gruppenspezifische Fehler (vgl. Snijders/Bosker 1999: 16 ff.). Angenommen wird, dass die guppenspezifischen Fehlerterme zwischen den Gruppen bedeutsam variieren - wäre dies nicht der Fall, wäre eine Standardregression (OLS-Regression) angemessen.

Mehrebenenmodell für Paneldaten. Mit der Verwendung von Mehrebenenmodellen für Wiederholungsmessungen ist es möglich, nicht nur die Veränderung der Kriteriums- 
variablen auf der Personenebene zu untersuchen, sondern diese Veränderung über die Zeitachse zu bestimmen (Langer 2004: 223). Die Besonderheit der Mehrebenenmodellierung bei der Analyse von Paneldatensätzen (wie z.B. des SOEP) besteht darin, dass die Beobachtungseinheiten der ersten und hierarchisch niedrigsten Ebene die Messzeitpunkte sind. Diese Ebene der Veränderungen enthält die im Zeitverlauf veränderbaren Variablen, wie z. B. das Erhebungsjahr, die Lebens- und Gesundheitszufriedenheit sowie das Alter und den Bildungsstand der Personen. Der Bildungsstand wurde in diese Ebene aufgenommen, um seine Dynamik vor allem im Hinblick auf nachgeholte sekundäre und tertiäre Bildungszertifikate zu berücksichtigen. Die zweite hierarchische Ebene stellen die Befragungspersonen selbst dar. Auf dieser Ebene werden die Variablen als stabil über den Erhebungszeitraum betrachtet; in ihr sind bestehende Charakteristika der Personen enthalten wie das Geburtsjahr bzw. die Kohortenzugehörigkeit.

Weitere Ebenen betreffen dann soziale Kontexte (z.B. Haushalte, Schulen, Regionen etc.). Die in den folgenden Modellen zum subjektiven Wohlbefinden im Zeitverlauf dritte und höchste Ebene enthält die Haushaltszugehörigkeit der Personen. Zwischen diesen unter- und übergeordneten Analyseeinheiten besteht ein Verhältnis der Inklusion, wie es mehrstufigen Zufallsauswahlen entspricht. In dieser Hinsicht ist das SOEP ein Datensatz, der für Mehrebenenmodelle mit drei Ebenen geeignet ist ${ }^{13}$ und diese aus der Perspektive des Mehrebenenansatzes sogar erfordert (vgl. Bryk/ Raudenbush 2002; Engel 1998: 113).

Mit der Mehrebenenanalyse ist es möglich, die Veränderungen von Einstellungen (hier die Lebenszufriedenheit) auf der Personenebene zu modellieren. Zunächst wird der Veränderungsprozess auf der Ebene der befragten Personen untersucht. Im Anschluss wird geprüft, welche zeitabhängigen bzw. zeitunabhängigen Personen- und Kontextmerkmale für eine Erklärung dieser differenziellen Veränderungsprozesse herangezogen werden können (vgl. Langer 2004: 298). Im Rahmen der folgenden Analysen werden die Mehrebenenmodelle als Hierarchisch Multivariate Lineare Modelle (HMLM) mit dem Programmpaket HLM (Bryk/Raudenbush 2002) geschätzt.

\section{Untersuchungsdesign: Datenbasis und Messinstrumente}

Datenbasis. Die Analyse der Veränderungen im subjektiven Wohlbefinden über einen längeren Zeitraum ermöglicht das Sozio-Oekonomische Panel (SOEP), eine seit 1984 jährlich vom Deutschen Institut für Wirtschaftsforschung (DIW) in Berlin durchgeführte Befragung per Wahrscheinlichkeitsauswahl gezogener Haushalte (Hanefeld 1987). Sowohl die Lebenszufriedenheit als auch die Zufriedenheit mit der Gesundheit gehörten bisher zu jedem Messzeitpunkt zum Fragenprogramm. Die folgenden Analysen basieren auf den kumulierten Panel-Befragungen von 1984 bis 2002. Um den Bildungshintergrund und den Sozialisationshintergrund sowie die gesellschaftlichen Ereignisse kontrollieren zu können, enthält die hier genutzte Nettogesamtstichprobe $(\mathrm{N}=16216)$ nur westdeutsche Befragte, die den Geburtsjahrgängen zwischen 1919 und 1963 angehören und zum Zeitpunkt des

13 Mehrebenenmodelle stellen hohe Anforderungen an die Daten (hierarchische Ordnung der Daten, ausreichende Fallzahl auf höhergeordneten Ebenen, bei längsschnittlichen Analysen: Panel-Daten) und die zu analysierende Stichprobe. 
Eintretens in das Panel nicht jünger als 21 Jahre alt waren. Die Reduktion der Stichproben auf die Kohorten zwischen 1919 und 1963 beugt Selektionseffekten vor. Die nach 1963 Geborenen würden in das Panel - Erhebungszeitraum 1984-2002 - erst hineinwachsen, hätten also schon von vornherein systematische Ausfälle in den ersten Erhebungsjahren. Geburtsjahre vor 1919 fielen durch die höhere Mortalität aus dem Panel heraus - dies auch wiederum systematisch, denn höher gebildete alte Menschen haben eine höhere Lebenserwartung und verbleiben länger im Panel (Becker 1998; Klein/Unger 2001).

Messinstrumente. Abhängige Variable der Analysen ist die Lebenszufriedenheit. Dieser Indikator wurde auf einer elfstufigen Skala erhoben. Die Befragten hatten sich bezüglich der Frage „Wie zufrieden sind Sie gegenwärtig, alles in allem, mit ihrem Leben?“ auf einem Kontinuum zwischen den Antwortmöglichkeiten ,ganz und gar unzufrieden“ (Wert 0) und ,ganz und gar zufrieden“ (Wert 10) einzuordnen. Diese an Campbell (1981) angelehnte Messmethode bezieht sich auf eine ,absolute“ Einschätzung des subjektiven Wohlbefindens, das heißt diese Vorgehensweise baut nicht explizit auf den Vergleich mit anderen Personen auf. Der Bildungsstand, erfragt nach der CASMIN-Klassifikation (Braun/Müller 1997) als Kombination aus höchstem erworbenen allgemeinbildenden und beruflichen Bildungszertifikat, wurde in eine diskrete Variable mit vier Ausprägungen überführt: In der niedrigsten Kategorie sind Personen ohne Abschluss und Personen mit Hauptschulabschluss ohne berufliche Bildung zusammengefasst (CASMIN 1a, 1b; $\mathrm{N}$ =2472). Der nächsthöheren Kategorie werden Hauptschüler mit Berufsausbildung und Personen mit einem Abschluss mittlerer Reife (mit oder ohne beruflicher Ausbildung) zugeordnet (CASMIN 1c, 2a, 2b; $\mathrm{N}$ = 9537). In der dritten Kategorie finden sich Abiturienten und Fachabiturienten mit und ohne Berufsausbildung (CASMIN 2c_gen, 2c_voc; $\mathrm{N}=1245$ ), während die höchste Kategorie den Individuen mit tertiären Bildungszertifikaten (Fachhochschule, Hochschule) vorbehalten bleibt (CASMIN 3a, 3b; N = 2962). Aus dem Geburtsjahr, das auch eigenständig als mittelwertszentrierte metrische Kohortenvariable in die multivariaten Analysemodelle eingeführt wird, wurden sechs KohortenDummyvariablen gebildet, die - mit Ausnahme der jüngsten Kohorte 1959-1963 - zehn Altersj ahrgänge umfassen und im Lichte der Bildungsexpansion interpretiert werden sollen. Entsprechend der These der kognitiven Mobilisierung von Baumert (1991; Baumert et al. 2003) sind die Generationen, die besonders von der Bildungsexpansion betroffen waren - die Geburtskohorten der 1949-1958 und der 1959-1963 Geborenen -, auf einem höheren Stand der kognitiven Mobilisierung. Im Hinblick auf Sozialisationsmerkmale (sozioökonomische Lage in der Phase der primären Sozialisation, Kriegsereignisse als besondere Stressoren; vgl. Becker 1998) ist auszuführen, dass diese beiden jüngsten Kohorten in relativem Wohlstand und frei von Kriegstraumata aufgewachsen sind.

Das Alter der Befragten zum Erhebungszeitpunkt wird als absolutes Alter in Jahren in die Modelle integriert. ${ }^{14}$ Die Periode kann infolge des Konfundierungsproblems der

14 Diese lineare Modellierung des Alterseffekts wird gewählt, da andere Verläufe - etwa u-förmige oder kurvilineare Verläufe - nicht eindeutig theoretisch abzuleiten sind. Dies gilt insbesondere vor dem Hintergrund der Ausklammerung des Gesundheitsaspekts. Das Alter bildet somit vor allem lebenszyklische Veränderungen ab, die nicht auf dem Gesundheitsaspekt beruhen, während die Gesundheitszufriedenheit die im Lebenszyklus auftretenden zunehmenden Gesundheitsprobleme thematisiert. 
A-P-K-Analyse nicht als zeitliche Variable eingeführt werden (vgl. Glenn 2005: 23). Das Erhebungsjahr wird daher durch die jeweilige Arbeitslosenquote der amtlichen Statistik für die alten Bundesländer ersetzt. Die Arbeitslosigkeit in Westdeutschland als Periodenvariable wird in den komplexen Modellen zudem durch die individuelle Arbeitslosigkeit zum Erhebungszeitpunkt (Dummy-Variable) kontrolliert, um sicherzustellen, dass der Effekt der Arbeitslosenquote nicht im Rahmen eines ökologischen Fehlschlusses missinterpretiert wird. Die Gesundheitszufriedenheit wurde - wie die Lebenszufriedenheit - auf einer elfstufigen Skala erfasst. Gefragt wurde „Wie zufrieden sind Sie heute mit Ihrer Gesundheit?“

\section{Untersuchungsergebnisse}

\subsection{Deskriptive Ergebnisse}

Die visuelle Inspektion der kohorten- und bildungsspezifischen Veränderung des subjektiven Wohlbefindens liefert erste Hinweise auf Effekte auf die Lebenszufriedenheit. Zunächst ist hinsichtlich des Ausmaßes an Zufriedenheit festzuhalten, dass sich die Befragten im Durchschnitt zu allen Messzeitpunkten eher zufrieden zeigen.

Abbildung 2: Bildungsspezifischer Wandel des subjektiven Wohlbefindens

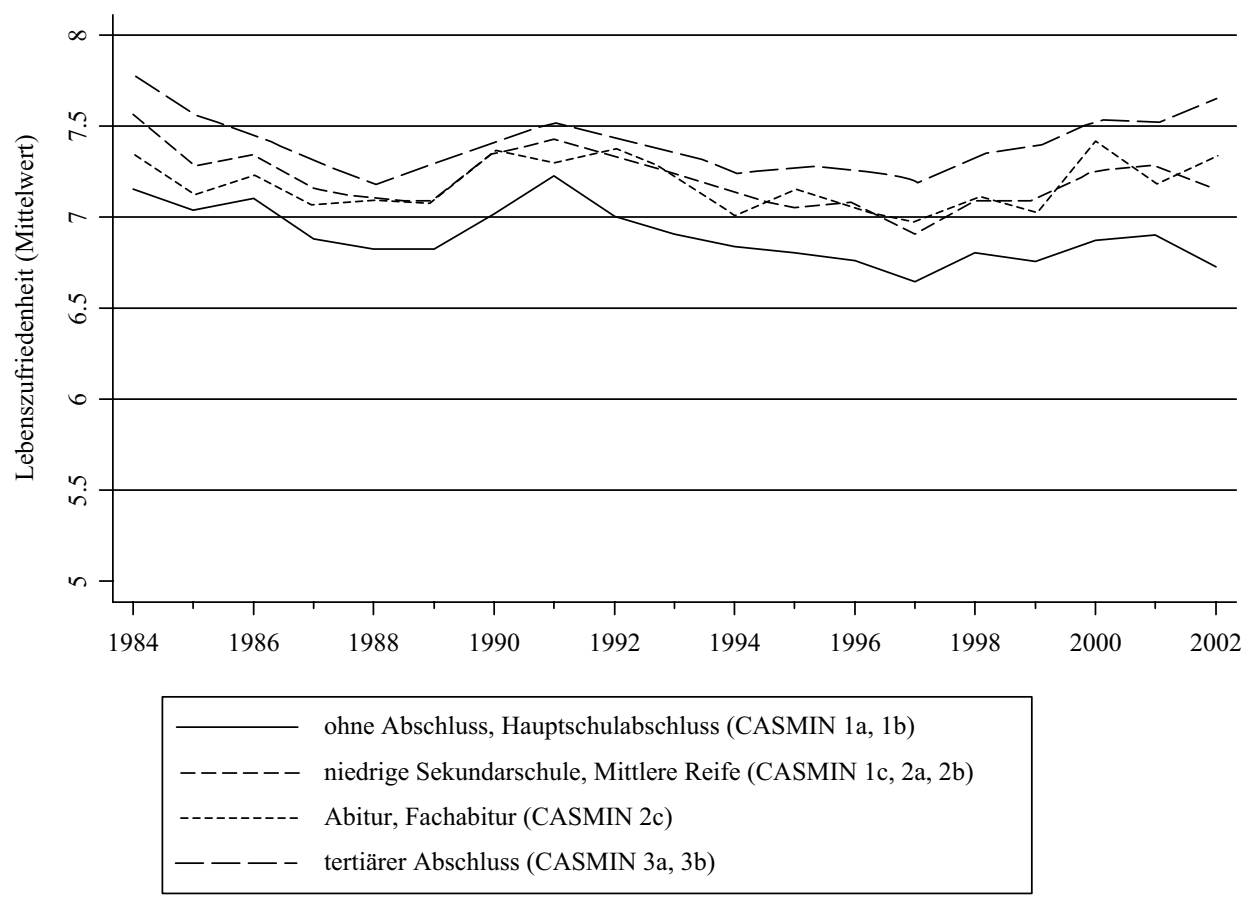

Quelle: SOEP, 1984-2002, eigene Berechnungen 


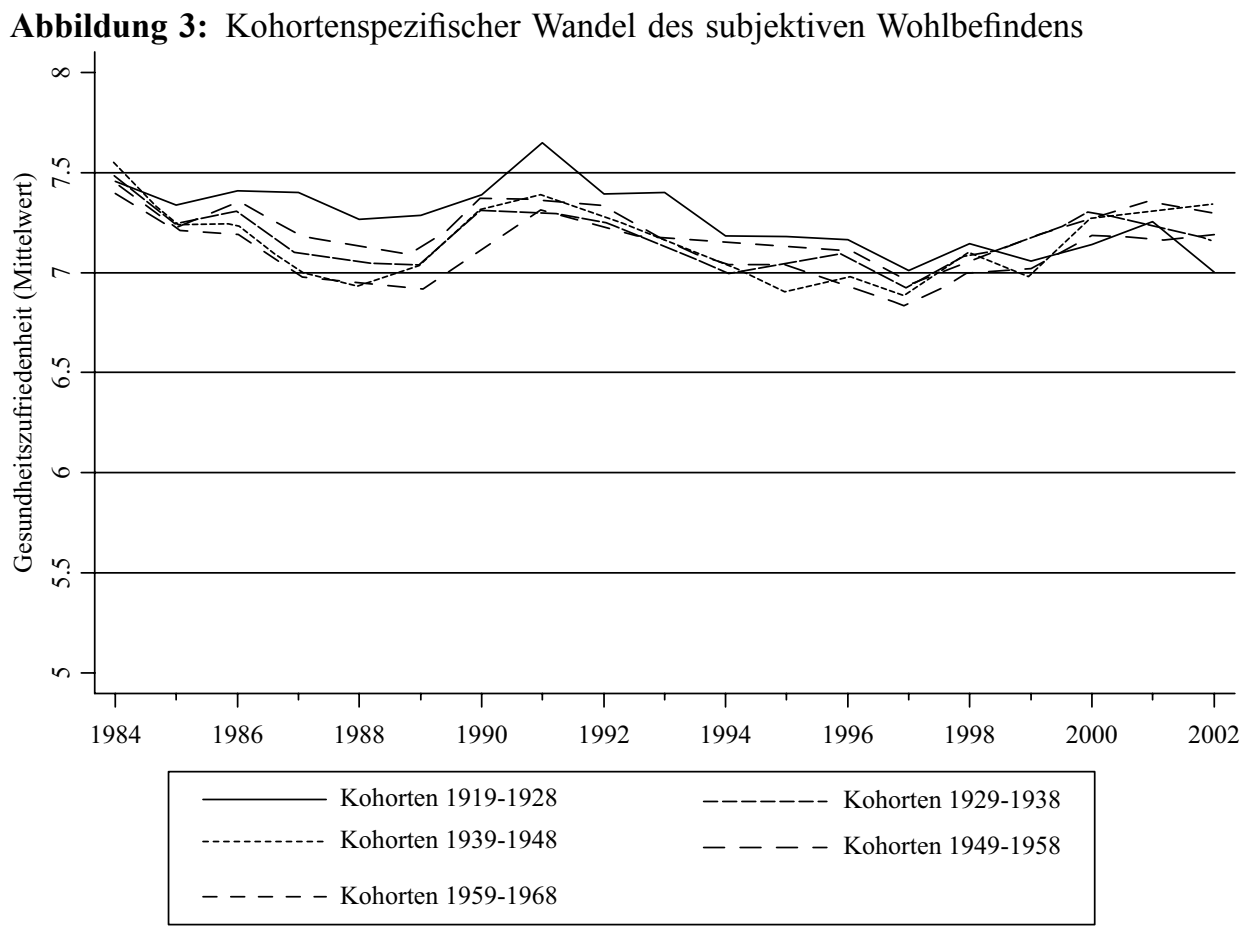

Quelle: SOEP, 1984-2002, eigene Berechnungen

In der bildungsspezifischen Betrachtung (Abbildung 2) sind Bildungsunterschiede in der Lebenszufriedenheit - höher Gebildete zeigen sich zufriedener - erkennbar. Individuen ohne Abschluss oder mit Hauptschulabschluss zeigen über alle Messzeitpunkte hinweg die geringste Lebenszufriedenheit, während diejenigen mit tertiärer Bildung durchweg die höchste Zufriedenheit angeben. Während die am niedrigsten Gebildeten über den Erhebungszeitraum zwischen 1984 und 2002 einen zunehmend größeren Abstand in der Lebenszufriedenheit zu den anderen Bildungsgruppen aufweisen, bleiben die sonstigen Bildungsunterschiede relativ konstant. Gleichzeitig zeigen sich geringe zeitliche Veränderungen, die für alle Kohorten ähnlich verlaufen. Dieser Befund weist auf leichte Periodeneffekte hin, die offenbar Ende der 1980er Jahre zu einer leichten Abnahme und Anfang der 1990er Jahre zu einer vorübergehenden Zunahme an Lebenszufriedenheit geführt haben. Nach einem leichten Absinken der Lebenszufriedenheit Mitte der 1990er Jahre ist danach wieder ein Ansteigen zu konstatieren.

Bei Betrachtung von Abbildung 3 sind kaum kohortenspezifische Unterschiede in der Lebenszufriedenheit zu erkennen. Einzig die älteste Kohorte zeigt zunächst geringfügig höhere Zufriedenheitswerte, die mit steigendem Alter abnehmen. Es sind wiederum im Beobachtungszeitraum von 1984 bis 2002 leichte Periodeneffekte identifizierbar: leichtes Absinken der Lebenszufriedenheit bis Ende der 1980er; Anstieg Anfang der 1990er; stetiges Absinken bis Mitte der 1990er und danach wieder eine Zunahme. 
Abbildung 4: Kohortenspezifischer Wandel der Gesundheitszufriedenheit

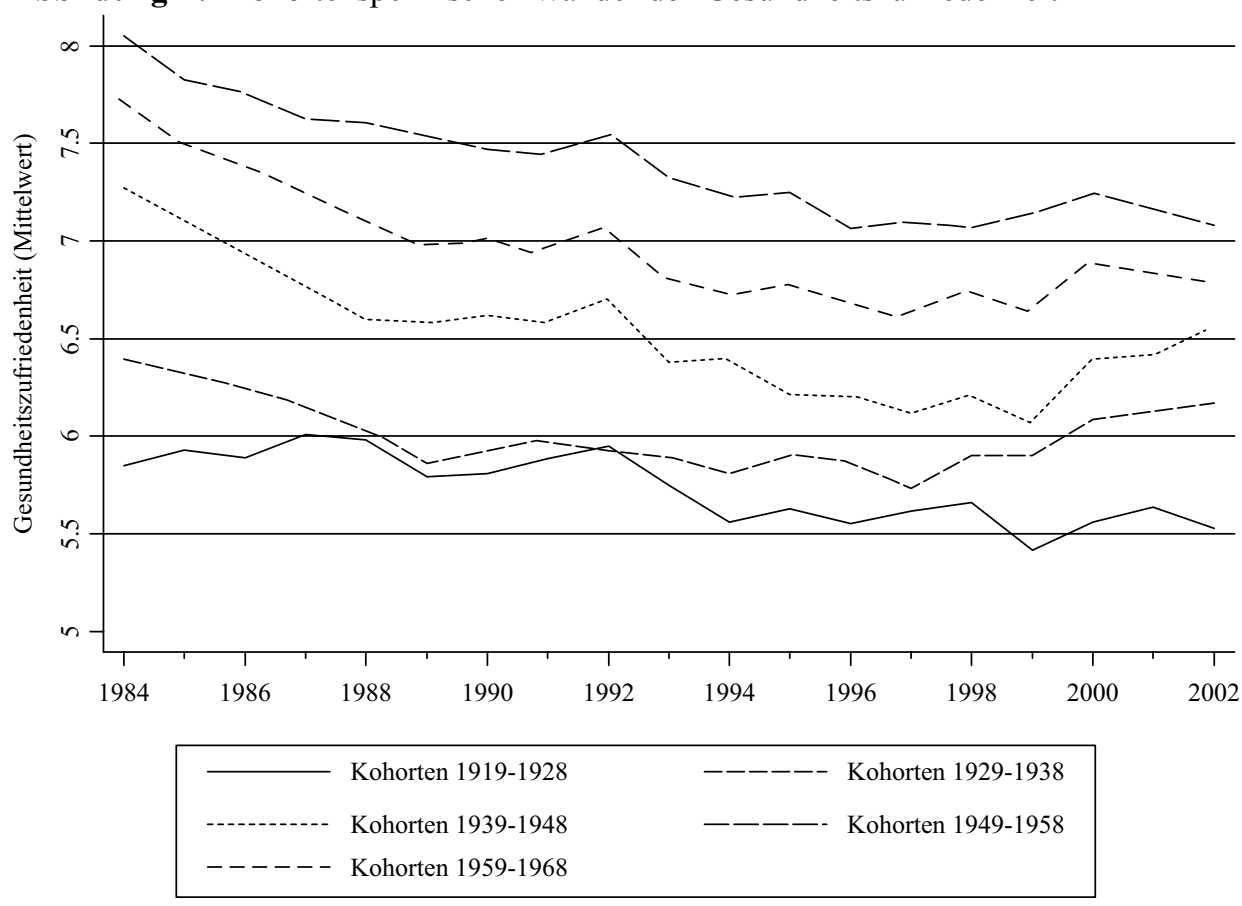

Quelle: SOEP, 1984-2002, eigene Berechnungen

Eine visuelle Inspektion der Gesundheitszufriedenheit (Abbildung 4) zeigt das erwartete deutliche Bild: Ältere Kohorten berichten eine niedrigere Gesundheitszufriedenheit. Darüber hinaus lässt sich aufgrund der Parallelität des Absinkens über den Zeitraum zwischen 1984 und 2002 ein starker Alterseffekt vermuten.

Die Arbeitslosenquote der alten Bundesländer - die als für das subjektive Wohlbefinden relevantes Periodencharakteristikum im Rahmen der A-P-K-Analysen die Periodenvariable ersetzen wird - war zwischen 1984 und 2002 Schwankungen unterworfen (Abbildung 5): Zunächst sinkt die Arbeitslosenquote zwischen 1984 und 1989 langsam ab, um dann vorübergehend anzusteigen. Zu Beginn der 1990er Jahre kommt es zu einer Entspannung des westdeutschen Arbeitsmarktes. Interessanterweise sind zur gleichen Zeit für die Lebenszufriedenheit besonders hohe Ausprägungen zu konstatieren. Mitte der 1990er Jahre steigt die Arbeitslosenquote wiederum an, um 1997 ein neues Rekordhoch zu erreichen. Bis zum Jahr 2001 geht die Arbeitslosenquote dann wieder leicht zurück. Ein Vergleich der Entwicklung der Lebenszufriedenheit mit der Entwicklung der Arbeitslosenquote lässt leichte kausale Zusammenhänge vermuten.

Die Tendenz zur Heterogenisierung der oberen Bildungsschichten, die in den theoretischen Überlegungen zur Rolle der Bildungsexpansion thematisiert wurde, ist in Abbildung 6 anhand der den Analysen zugrunde liegenden Daten grafisch umgesetzt. Es zeigt sich, dass der Effekt der Heterogenisierung - erkennbar an der Annäherung an niedriger Gebildete - insbesondere bei denjenigen mit Abitur anzutreffen ist, die keinen 
Abbildung 5: Arbeitslosenquote Westdeutschland (Arbeitslosenquote in \% aller abhängig beschäftigten zivilen Erwerbspersonen)

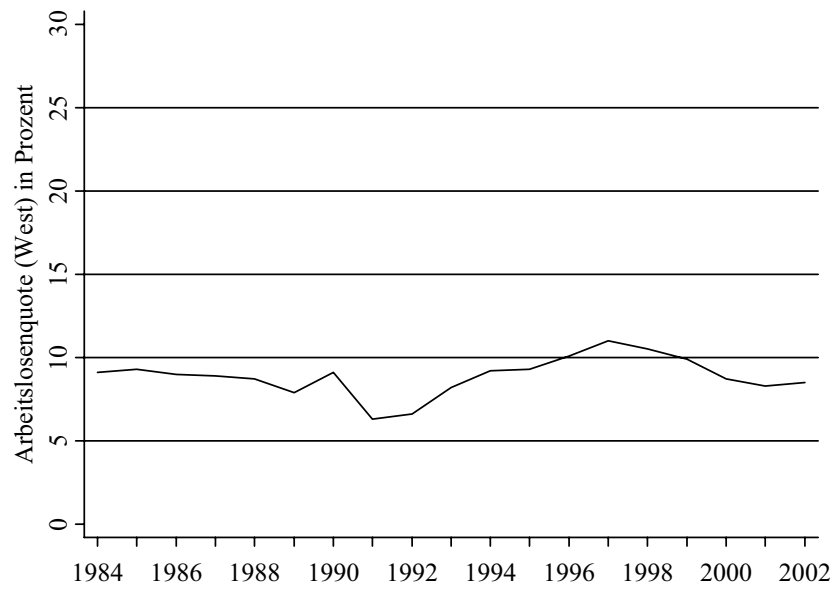

Quelle: Statistisches Bundesamt/IAB

Abbildung 6: Interaktionseffekt Kohorte x Bildung

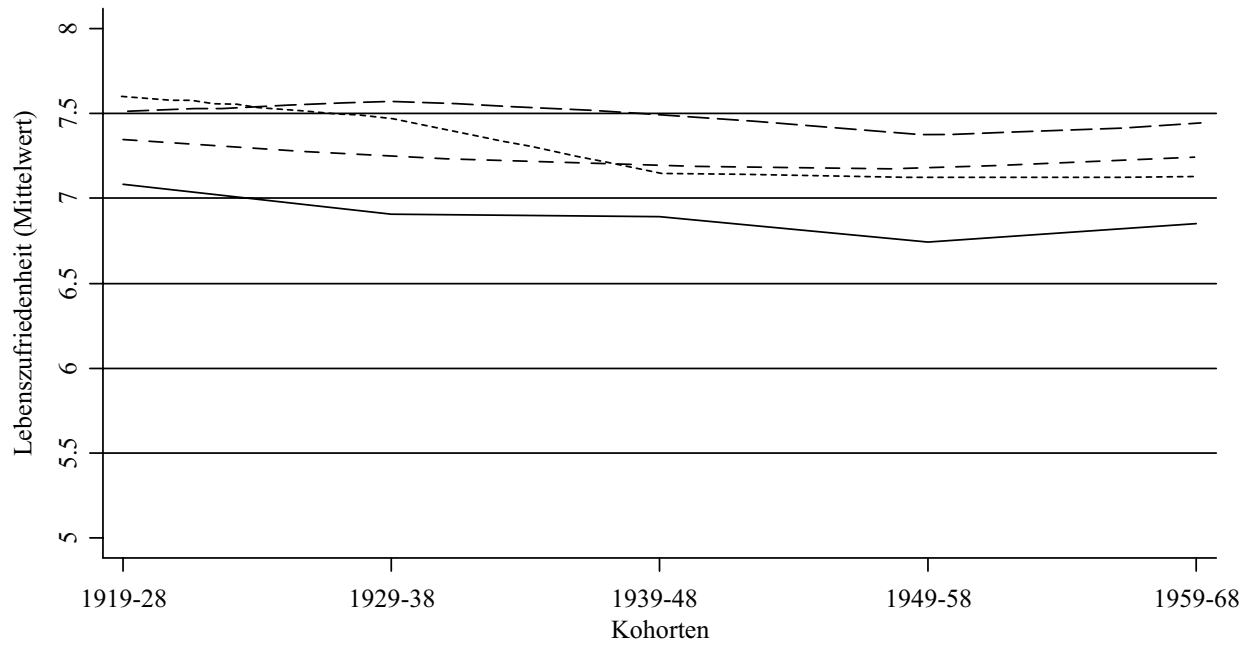

ohne Abschluss, Hauptschulabschluss (CASMIN 1a, 1b)

niedrige Sekundarschule, Mittlere Reife (CASMIN 1c, 2a, 2b)

Abitur, Fachabitur (CASMIN 2c)

tertiärer Abschluss (CASMIN 3a, 3b)

Quelle: SOEP, 1984-2002, eigene Berechnungen 


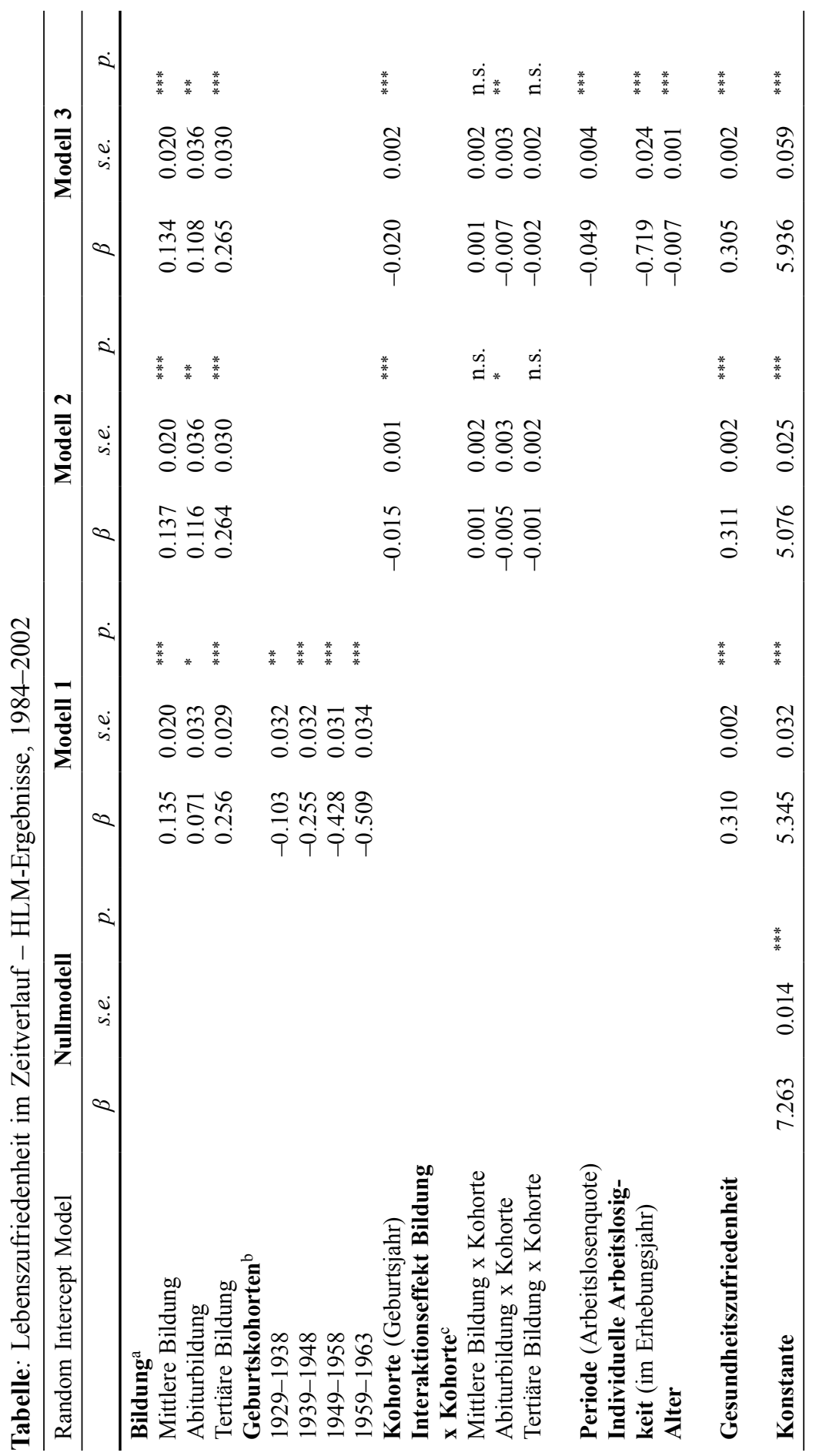




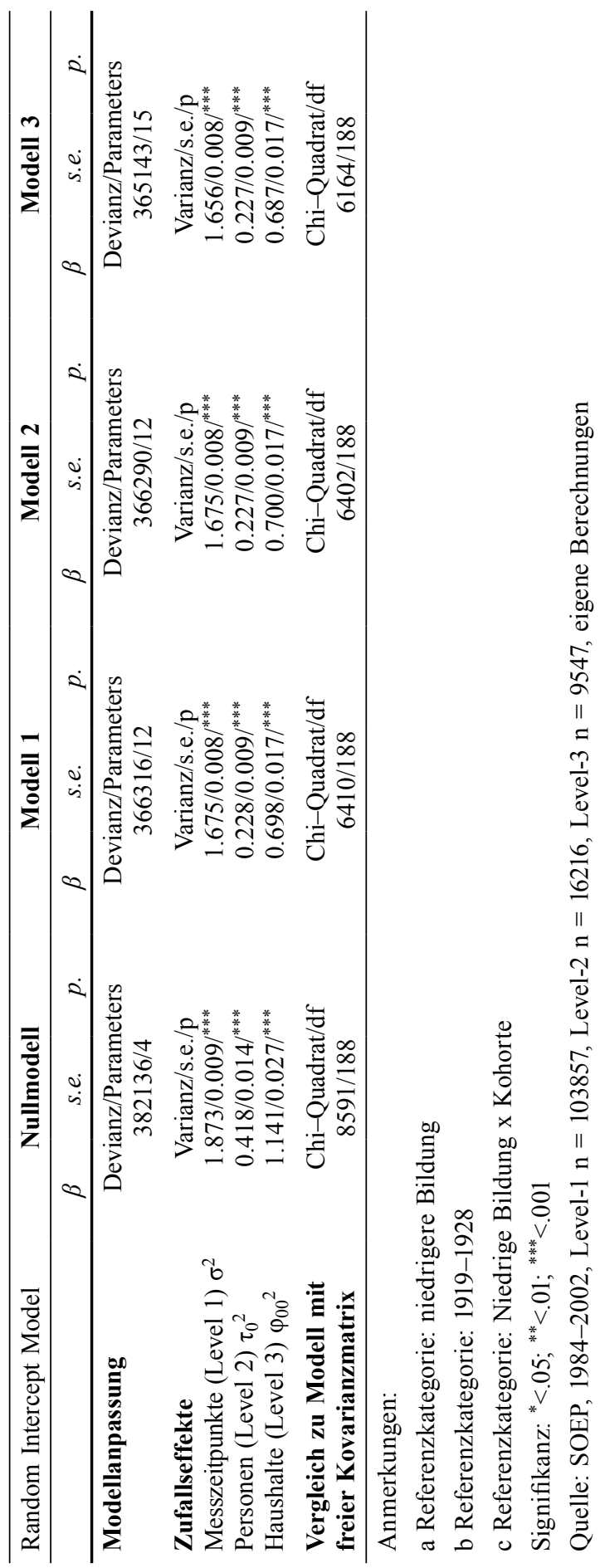


weiteren Tertiärabschluss erworben haben, während die positive Distinktion der Hochschulabsolventen im Hinblick auf die Lebenszufriedenheit über alle Kohorten erhalten bleibt. Die distinkte Stellung der sogenannten Kellerkinder der „Bildungsexpansion“ (Klemm 1991), die über keinen Abschluss oder einen Hauptschulabschluss verfügen (Solga/Wagner 2001) und besonders unzufrieden mit ihrem Leben sind, erweist sich über die Kohortensukzession als relativ konstant.

Die visuelle Inspektion gibt erste Hinweise auf Bildungs-, Kohorten-, Alters- und Periodeneffekte. $\mathrm{Ob}$ die einzelnen Effekte genuin oder auf einen oder mehrere andere Effekte zurückzuführen und wie stark diese Effekte sind, kann nur in multivariaten A-PK-Analysen geklärt werden (vgl. Bürklin/Klein/Ruß 1994: 595). Dies gilt insbesondere für die Frage, ob und inwieweit die sich in der visuellen Inspektion abzeichnenden Kohortenunterschiede auf Alterseffekte zurückgehen.

\subsection{Multivariate Ergebnisse: HLM-Modelle der Lebenszufriedenheit}

Die Mehrebenenmodelle unter Berücksichtigung von Alters-, Perioden- und Kohorteneffekten werden schrittweise berechnet und dargestellt, um genauere Auskunft über die genuinen Effekte der einzelnen Variablen zu geben.

Die Schätzmodelle zur Erklärung der Lebenszufriedenheit in Abhängigkeit von unterschiedlichen Zeitdimensionen sind in der Tabelle dargestellt. Das Ausgangsmodell (Nullmodell) ist das als Referenzmodell zur Berechnung der erklärten Varianz dienende Intercept-only-Modell, das die Schätzungen der Standardfehler für Mittelwerte und Mittelwertsdifferenzen der abhängigen Variablen und die Fehlervarianzen auf den Ebenen der Messzeitpunkte, der Befragten und der Haushalte enthält (Interzept der Lebenszufriedenheit $M=7.263$ ). Aus dem Verhältnis der Gesamtvarianz zu den einzelnen Varianzkomponenten ergibt sich die jeweilige Intraklassen-Korrelation: Auf Differenzen zwischen den Messzeitpunkten innerhalb der Befragungspersonen sind 54,57\% der insgesamt zu beobachtenden Varianz zurückzuführen, 12,18\% auf Unterschiede zwischen den Befragungspersonen und 33,25\% auf Unterschiede zwischen den Haushalten. ${ }^{15}$

In Modell 1 werden die Bildung und die Kohorte als Personenmerkmale - unter Kontrolle der Gesundheitszufriedenheit - eingeführt. Es zeigt sich eine bedeutsame Beziehung zwischen Bildung und Lebenszufriedenheit: Höher Gebildete haben eine signifikant höhere Lebenszufriedenheit, wobei Personen auf tertiärem Bildungsniveau die höchste Lebenszufriedenheit aufweisen. Die Effekte der Kohorten-Dummyvariablen sind durchgehend signifikant. In der Kohortenabfolge sinkt die Lebenszufriedenheit mit jeder Kohorte ab: Die jüngeren Kohorten sind unzufriedener, als dies in Anbetracht ihres höheren Bildungsniveaus und ihrer höheren Gesundheitszufriedenheit zu erwarten wäre. Die Gesundheitszufriedenheit zeigt den erwarteten starken positiven Effekt auf die Lebenszufriedenheit.

In Modell 2 werden die Kohorten in einer metrischen Operationalisierung, Geburtsjahr (mittelwertszentriert), integriert. Dieser Kohorteneffekt erweist sich - wie in Modell 1 - als negativ. Die neu ins Modell aufgenommenen Interaktionsterme aus Bildung

15 Bei der Interpretation der Intraklassen-Korrelation ist die geringe durchschnittliche Haushaltsgröße - im Schnitt leben 1,7 Personen in den untersuchten Haushalten - zu berücksichtigen. 
und Kohorte ${ }^{16}$ stützen die These der Heterogenisierung der Bildungsschichten nach sozialer Herkunft nur teilweise, denn nur diejenigen mit Abiturbildung ohne weiterführenden Abschluss nähern sich über die Kohortenabfolge in ihrer Lebenszufriedenheit tendenziell den niedriger Gebildeten an. Die Kluft in der Lebenszufriedenheit zwischen gering Gebildeten (ohne Abschluss, Hauptschulabschluss ohne Ausbildung) und höher Gebildeten bleibt über die Bildungsexpansion jedoch erhalten.

Unter Hinzufügung des Alters und der Arbeitslosenquote (Periode) sowie der individuellen Arbeitslosigkeit in Modell 3 bleibt der negative Einfluss der Kohorte konstant: Später Geborene sind unzufriedener als ältere Geburtskohorten. Auch unter simultaner Betrachtung des Einflusses des Alterns gilt, dass später Geborene unter Berücksichtigung ihres jüngeren Alters eine vergleichsweise niedrige Lebenszufriedenheit haben. Die Arbeitslosenquote als Periodenmerkmal zeigt sich unter gleichzeitiger Kontrolle der individuellen Arbeitslosigkeit im Erhebungsjahr als signifikanter Prädiktor der Lebenszufriedenheit. Die befragten Individuen berichten in Jahren höherer Arbeitslosigkeit eine insgesamt geringere Zufriedenheit mit ihrem Leben. Die individuelle Arbeitslosigkeit beeinträchtigt jedoch - im Hinblick auf die Effektgröße - die Lebenszufriedenheit ungleich stärker. Das Alter ist ebenfalls ein signifikanter Einflussfaktor: Mit zunehmendem Alter sinkt die Lebenszufriedenheit leicht. Die Gesundheitszufriedenheit behält ihren substanziellen Einfluss auf das Ausmaß der Lebenszufriedenheit. Bezüglich der Modellgüte weist das in Modell 3 im Vergleich zu den vorherigen Modellen niedrigere Devianz-Maß auf eine Verbesserung der Erklärungskraft hin.

\section{Diskussion}

In der Gesamtbetrachtung scheint zunächst der bereits in vielen Studien (z.B. Mohr 1987: 167; Glatzer 1985) konstatierte Befund auf, dass die Westdeutschen eine relativ hohe Lebenszufriedenheit haben. Im Hinblick auf die inhaltlichen Fragestellungen ist zu konstatieren, dass die Lebenszufriedenheit - unter Kontrolle des Effekts der Gesundheitszufriedenheit - stark vom Bildungsniveau abhängig ist. Hypothesenkonform zeigten höher Gebildete (Hypothese 1) eine höhere Lebenszufriedenheit, was auf erweiterte kognitive Fähigkeiten, Kompetenzen und Möglichkeiten zur Bedürfnisbefriedigung schließen lässt. Bildung kann dabei in Anlehnung an Becker (1998) sowohl als Humanvermögen (Fähigkeiten) als auch als Humankapital (ökonomische Ressourcen) gedeutet werden. Die Kohorteneffekte sind Ausdruck verschiedener Einflüsse: Zunächst ist auszuführen, dass ein Teil der Kohortenunterschiede im subjektiven Wohlbefinden auf den kohortenspezifischen, mit der Bildungsexpansion ansteigenden Bildungsniveaus beruht. ${ }^{17}$ In den komplexen A-P-K-Modellen sind entgegen Hypothese 2 jüngere bzw. später geborene Kohorten offenbar unzufriedener mit ihrem Leben. Der Effekt der

16 Die Interaktionseffekte wurden - zur Senkung der bei Einführung von Interaktionseffekten zunehmenden Multikollinearität (vgl. Jaccard/Turrisi/Wan 1990) - jeweils aus einer Dummyvariablen des Bildungsniveaus und der mittelwertszentrierten Variablen „Geburtsjahr“ gebildet. Referenzkategorie ist der Effekt „niedrige Bildung x Kohorte“.

17 In hier nicht dargestellten Analysen reduzierte sich bei Einschluss der Kohorteneffekte der Bildungseffekt. 
Höherbildung im Zuge der Bildungsexpansion wird vermutlich überlagert von gesellschaftlichen Problemen wie der Ausbildungsproblematik und dem Arbeitsplatzmangel, die die Möglichkeiten der Bedürfnisbefriedigung einschränken. Aus theoretischer Sicht könnte der negative Effekt des Geburtsjahrs so gedeutet werden, dass die zu früheren Zeitpunkten Geborenen in Anbetracht ihres zunehmenden Alters - und der damit verbundenen Gesundheitsprobleme - offenbar mit ihrem Leben zufriedener sind als die später Geborenen (vgl. Schneider 1999). Von besonderem Interesse sind die Interaktionseffekte aus Bildung und Kohorte (Geburtsjahr), welche dem in Hypothese 3 postulierten Heterogenisierungseffekt entsprechen. Während über die Kohortenabfolge die Abstände in der Lebenszufriedenheit zwischen gering Gebildeten, Personen mit Mittlerer Reife und den tertiär Gebildeten gleich bleiben, nähern sich die Abiturienten ohne tertiären Abschluss in ihrer Lebenszufriedenheit tendenziell den niedrig Gebildeten an. Die negative Distinktion der „Kellerkinder“ der Bildungsexpansion bleibt über die Kohortenabfolge somit erhalten, das heißt sie sind weiterhin unzufriedener als alle anderen Bildungsgruppen und konnten sich somit auch bezüglich des subjektiven Wohlbefindens nicht im Zuge der Bildungsexpansion verbessern (Solga/Wagner 2001). Im Fazit kann geschlossen werden, dass sich offenbar im Rahmen dieser Interaktionseffekte die durch die Bildungsreform verursachte Heterogenisierung der höheren Bildungsschicht wie sie Baumert (1991) thematisiert - und die damit verbundene Annäherung der höher Gebildeten an die niedrig Gebildeten bezüglich ihrer Fähigkeiten und Kompetenzen nur im Hinblick auf die Abiturienten nachweisen lässt, die keine Hochschule oder Fachhochschule besucht haben. In Widerlegung der Hypothese 4, in der kein Effekt des Alters auf die Lebenszufriedenheit angenommen wurde, ist ein sehr geringer negativer Alterseffekt auf die Lebenszufriedenheit zu konstatieren, was vermutlich mit der geringeren sozialen Unterstützung im Alter zu erklären ist. ${ }^{18}$ Die von den Befragten berichtete Lebenszufriedenheit war - entsprechend Hypothese 5 - in Jahren geringerer Arbeitslosigkeit signifikant höher; dies unterstreicht die Bedeutsamkeit dieses Makroebenen-Charakteristikums für die subjektive Wahrnehmung (Terwey 1990). Einen ähnlichen Effekt hat die individuelle Arbeitslosigkeit zum jeweiligen Erhebungszeitpunkt. Somit sind Makro- und Mikroeffekt gleichgerichtet; ein ökologischer Fehlschluss liegt offenbar nicht vor. Es kann nun vermutet werden, dass die Perzeption der Arbeitslosenquote die Lebenszufriedenheit trübt, die individuelle Arbeitslosigkeitserfahrung aber letztlich entscheidend ist. Die Gesundheitszufriedenheit hatte schließlich entsprechend der Vermutung (vgl. Dehlinger/Ortmann 1992) einen starken positiven Einfluss auf die Lebenszufriedenheit.

Die Befunde sind vor dem Hintergrund inhaltlicher Limitierungen zu interpretieren. Die individuelle Zufriedenheit ist abhängig von Vergleichsmaßstäben, welche Bedürfnisse bestehen, zu welchem Grad diese erfüllt sein müssen und im Vergleich zu wem der Grad der Bedürfnisbefriedigung bestimmt wird. Das hier angedeutete Phänomen ist das der relativen Deprivation, der Wahrnehmung einer Differenz zwischen den

18 In hier nicht dargestellten Analysen zeigt sich ein noch stärkerer negativer Alterseffekt, wenn die Gesundheitszufriedenheit nicht kontrolliert wird. Der Gesundheitsaspekt hat somit insbesondere in höheren Lebensaltern eine besondere Bedeutung für die Einschätzung der Lebenszufriedenheit. 
Ressourcen einer Person und den Ressourcen, über die sie nach eigenem Eindruck auch im Hinblick auf Vergleichsgruppen - verfügen sollte (vgl. u. a. Runciman 1966; Gurr 1970). Die individuellen Präferenzen, das Anspruchsniveau und die Vergleichsgruppen konnten jedoch in den Analysen nicht berücksichtigt werden. Aus ähnlichem Blickwinkel erscheint eine Gegenüberstellung objektiver und subjektiver Indikatoren sinnvoll, da objektiv identische Situationen und Lebensumstände von verschiedenen Personen unterschiedlich wahrgenommen und interpretiert werden (Noll 1989: 26). ${ }^{19} \mathrm{Im}$ Hinblick auf den Bildungseffekt ist zu fragen, inwieweit mit einem höheren Bildungsniveau nicht auch höhere Standards bzw. Ansprüche sowie spezifische (bildungshomogene) Bezugsgruppen-Umwelten (vgl. Blossfeld/Timm 2003) verbunden sind. Denkbar wäre, dass die höher Gebildeten infolge eines höheren Anspruchniveaus, welches u.a. auf besser gestellte Bezugsgruppen zurückgeht, eine geringere Lebenszufriedenheit haben sollten, als dies ihre bessere Stellung und ihre bessere Ressourcenausstattung vermuten ließen. Im Rahmen der hier vorliegenden Studie zeigten sich die höher Gebildeten dennoch signifikant zufriedener als die niedriger Gebildeten. Insofern ist im Hinblick auf die Überlegungen zum Anspruchsniveau und den Bezugsgruppen davon auszugehen, dass die Lebenszufriedenheit der höher Gebildeten sogar tendenziell unterschätzt wurde und der Bildungseffekt noch stärker sein sollte. Darüber hinaus könnte in Anschlussanalysen die Variable Geschlecht, die hier aus Gründen der Komplexitätsreduktion ausgeklammert wurde, Berücksichtigung finden. Es wird angenommen, dass sich geschlechtsspezifische Strategien der Lebensführung auf das subjektive Wohlbefinden auswirken. Zudem erscheint eine adäquate Modellierung kritischer Lebensereignisse als sinnvoll.

Vor einer abschließenden methodischen Bewertung der HLM-Modellierung ist auf einige andere methodische Aspekte hinzuweisen. Erstens könnte die Frage aufgeworfen werden, ob sich hinter den Veränderungen in der Zeit bzw. der Kohortenabfolge tatsächlich die Bildungsexpansion verbirgt oder ob nicht weitere gesellschaftliche Prozesse etwa der medizinische Fortschritt - für die Veränderungen des subjektiven Wohlbefindens verantwortlich sind. Dies kann im Rahmen dieser empirischen Studie nicht geklärt werden; eine quantitative Operationalisierung medizinischen Fortschritts fällt generell schwer. Eine Scheinkorrelation kann ausgeschlossen werden, denn die (positiven) Zusammenhänge zwischen Bildungsniveau und Lebenszufriedenheit auf der Individualebene sprechen klar für einen Zusammenhang zwischen Bildung und subjektivem Wohlbefinden. Zweitens könnten die HLM-Schätzungen im Random-effects-Modell durch eine eventuelle Korrelation der Individueneffekte mit den unabhängigen Modellvariablen verzerrt sein (Endogenität). Das Risiko einer verzerrten Koeffizientenschätzung wurde parallel zu diesen Analysen durch eine robuste Varianzschätzung (OLS-Regression) ausgeräumt. Drittens sind die Messinstrumente nochmals zu thematisieren. Es wurde nach der subjektiven Einschätzung der Lebens- und Gesundheitszufriedenheit gefragt. Das schließt, wie bei Einschätzungs- oder Meinungsfragen im Allgemeinen, Effekte sozialer Erwünschtheit nicht aus. Es könnte Anreize dafür geben, dass sich Befragte in sozialen Situationen zufriedener präsentieren, als sie es in Wirklichkeit sind (vgl. Noll

19 Ein interessantes Modell hierzu legen Glatzer und Zapf (1984a: 23) vor (Stichwort: Zufriedenheitsparadox versus Wohlfahrtsdilemma). 
1989: 32 ff.). Wie Scott und Nolan (2005) anmerken, geben die meisten Menschen auf die Frage nach dem Befinden ,fairly happy“ an.

Als Fazit zeigt sich bei aller Komplexität der Befunde, dass eine höhere Bildung ein Garant für eine höhere Lebenszufriedenheit ist. Mit der Anhebung des allgemeinen Bildungsniveaus zeichnet sich somit ein steigendes gesellschaftliches Zufriedenheitsniveau ab.

\section{Literatur}

Abel, Thomas (1999): Gesundheitsrelevante Lebensstile: Zur Verbindung von handlungs- und strukturtheoretischen Aspekten in der modernen Ungleichheitsforschung. In: Christoph Maeder/Claudine Burton-Jeangros/Mary Haour-Knipe (Hrsg.), Gesundheit, Medizin und Gesellschaft. zur Beiträge Soziologie der Gesundheit. Zürich: Seismo, S. 43-61.

Andrews, Frank M./Stephen B. Withey (1976): Social Indicators of Well-Being. New York: Plenum.

Argyle, Michael (1987): The Psychology of Happiness. London: Routledge.

Baier, Dirk/Klaus Boehnke (2007): Subjektives Wohlbefinden im Kulturvergleich. In: Gisela Trommsdorff/Hans-Joachim Kornadt (Hrsg.), Enzyklopädie der Psychologie. Kulturvergleichende Psychologie. Band 3: Anwendungsfelder der kulturvergleichenden Psychologie. Göttingen: Hogrefe, S. 463-488.

Baumert, Jürgen (1991): Langfristige Auswirkungen der Bildungsexpansion. In: Unterrichtswissenschaft 19, S. 333-349.

Baumert, Jürgen/Cordula Artelt/Eckhard Klieme/Michael Neubrand/Manfred Prenzel/Ulrich Schiefele/Wolfgang Schneider/Gundel Schümer/Klaus-Jürgen Tillmann/Manfred (Hrsg.) (2003): Pisa 2000. Ein differenzierter Blick auf die Länder der Bundesrepublik Deutschland. Opladen: Leske + Budrich.

Becker, Gary S. (1974): Human Capital. New York: Columbia University Press.

Becker, Rolf (1998): Bildung und Lebenserwartung in Deutschland. Eine empirische Längsschnittuntersuchung aus der Lebensverlaufsperspektive. In: Zeitschrift für Soziologie 27, S. 133-150.

Blanchflower, David G./Andrew J. Oswald (2004): Well-Being over Time in Britain and the USA. In: Journal of Public Economics 88, S. 1359-1386.

Blien, Ulrich/Michael Wiedenbeck (2002): Mehrebenenanalyse. In: Gerhard Kleinherz (Hrsg.), IAB-Kompendium Arbeitsmarkt- und Berufsforschung. Beiträge zur Arbeitsmarkt- und Berufsforschung, BeitrAB 250. Nürnberg: IAB, S. 309-324.

Blossfeld, Hans-Peter/Andreas Timm (2003): Educational Systems as Marriage Markets in Modern Societies: A Conceptual Framework. In: Hans-Peter Blossfeld/Andreas Timm (Hrsg.), Who Marries Whom? Educational Systems as Marriage Markets in Modern Societies. Dordrecht/Boston/London: Kluwer, S. 1-18.

Borchelt, Markus/Reiner Gilberg/Ann Horgas/Bernhard Geiselmann (1996): Zur Bedeutung von Krankheit und Behinderung im Alter. In: Paul Baltes/Karl-Ulrich Mayer (Hrsg.), Die Berliner Altersstudie. Berlin: Akademie Verlag, S. 449-474.

Bradburn, Norman M. (1969): The Structure of Psychological Well-Being. Chicago: Aldine Publishing Company.

Braun, Michael/Walter Müller (1997): Measurement of Education in Comparative Research. In: Comparative Social Research 16, S. 163-201.

Bryk, Anthony S./Stephen W. Raudenbush (2002): Hierarchical Linear Models. Applications and Data Analysis Methods. Newbury Park, CA: Sage Publications. 
Bürklin, Wilhelm/Markus Klein/Achim Ruß (1994): Dimensionen des Wertewandels. Eine empirische Längsschnittanalyse zur Dimensionalität und der Wandlungsdynamik gesellschaftlicher Wertorientierungen. In: Politische Vierteljahresschrift 35, S. 579-606.

Büschges, Günther/Martin Abraham/Walter Funk (1996): Grundzüge der Soziologie. München/ Wien: Oldenbourg.

Campbell, Angus (1981): The Sense of Well-Being in America. New York: McGraw-Hill.

Clark, Andrew/Andrew Oswald (1994): Unhappiness and Unemployment. In: The Economic Journal 104, S. 648-659.

Coleman, James S. (1991): Grundlagen der Sozialtheorie. Band 1: Handlungen und Handlungssysteme. München: Oldenbourg.

De Leeuw, Jan (2002): Series Editor's Introduction to Hierarchical Linear Models. In: Anthony S. Bryk/Stephen W. Raudenbush (Hrsg.), Hierarchical Linear Models. Applications and Data Analysis Methods. Newbury Park, CA: Sage Publications, S. xix-xxii.

Dehlinger, Erwin/Karlheinz Ortmann (1992): Gesundheitszufriedenheit in der Bundesrepublik Deutschland und der ehemaligen DDR - ein Vergleich. In: Das Gesundheitswesen 54, S. 88-94.

Diener, Ed/Randy J. Larsen (1984): Temporal Stability and Cross-situational Consistency of Affective, Cognitive, and Behavioral Responses. In: Journal of Personality and Social Psychology 47, S. 871-883.

Diener, Ed/Shigehiro Oishi/Richard E. Lucas (2003): Personality, Culture, and Subjective WellBeing. In: Annual Review of Psychology 54, S. 403-425.

Di Tella, Rafael/Robert J. MacCulloch/Andrew J. Oswald (2001): Preferences over Inflation and Unemployment. Evidence from Surveys of Happiness. In: American Economic Review 91, S. 335-341.

Engel, Uwe (1998): Einführung in die Mehrebenenanalyse. Grundlagen, Auswertungsverfahren und praktische Beispiele. Opladen/Wiesbaden: Westdeutscher Verlag.

Ferraro, Kenneth (1980): Self-ratings of Health among the Old and the Old-Old. In: Journal of Health and Social Behavior 21, S. 377-383.

Festinger, Leon (1957): A Theory of Cognitive Dissonance. Stanford: Stanford University Press.

Frey, Bruno S./ Alois Stutzer (2002): What Can Economists Learn from Happiness Research? In: Journal of Economic Literature 40, S. 402-435.

Geiger, Theodor (1987): Die soziale Schichtung des deutschen Volkes. Stuttgart: Enke.

Glatzer, Wolfgang (1984): Determinanten subjektiven Wohlbefindens. In: Wolfgang Glatzer/Wolfgang Zapf (Hrsg.), Lebensqualität in der Bundesrepublik. Frankfurt a.M./New York: Campus, S. 307-322.

Glatzer, Wolfgang (1985): Komponenten des Wohlbefindens. In: Statistisches Bundesamt (Hrsg.), Datenreport 1985. Bonn: Statistisches Bundesamt, S. 370-376.

Glatzer, Wolfgang/Wolfgang Zapf (1984a): Die Lebensqualität der Bundesbürger. In: Aus Politik und Zeitgeschichte B44, S. 3-25.

Glatzer, Wolfgang/Wolfgang Zapf (1984b) (Hrsg.): Lebensqualität in der Bundesrepublik. Objektive Lebensbedingungen und subjektives Wohlbefinden. Frankfurt a.M./New York: Campus.

Glenn, Norval D. (2005): Cohort Analysis. Thousand Oaks: Sage.

Grob, Alexander (1995): Subjective Well-Being and Significant Life Events across the Life Span. In: Swiss Journal of Psychology 54, S. 3-18.

Gurr, Ted R. (1970): Why Men Rebel? Princeton: Princeton University Press.

Hadjar, Andreas (2006): Bildungsexpansion und Wandel von sozialen Werten. In: Andreas Hadjar/ Rolf Becker (Hrsg.), Die Bildungsexpansion. Erwartete und unerwartete Folgen. Wiesbaden: VS Verlag für Sozialwissenschaften, S. 205-230.

Hadjar, Andreas/Rolf Becker (2006): Bildungsexpansion und Wandel des politischen Interesses in Westdeutschland zwischen 1980 und 2002. In: Politische Vierteljahresschrift 47, S. 12-34. 
Hanefeld, Ute (1987): Das Sozio-ökonomische Panel. Grundlagen und Konzeption. Frankfurt a.M./New York: Campus.

Hedström, Peter/Richard Swedberg (Hrsg.) (1998): Social Mechanisms. An Analytical Approach to Social Theory. Cambridge: Cambridge University Press.

Inglehart, Ronald (1997): Modernization and Postmodernization. Cultural, Economic, and Political Change in 43 Countries. Princeton: Princeton University Press.

Inglehart, Ronald/Hans-Dieter Klingemann (2000): Genes, Culture, Democracy, and Happiness. In: Ed Diener (Hrsg.), Culture and Subjective Well-Being. Cambridge: MIT Press, S. $165-184$.

Jaccard, James J./Robert Turrisi/Choi K. Wan (1990): Interaction Effects in Multiple Regression. Thousend Oaks/London/New Delhi: Sage.

Klecka, William R. (1971): Applying Political Generations to the Study of Political Behavior. A Cohort Analysis. In: Public Opinion Quarterly 35, S. 358-373.

Klein, Markus/Michaela Pötschke (2004): Die intraindividuelle Stabilität gesellschaftlicher Wertorientierungen. Eine Mehrebenenanalyse auf der Grundlage des Sozio-oekonomischen Panels (SOEP). In: Kölner Zeitschrift für Soziologie und Sozialpsychologie 56, S. 432-456.

Klein, Thomas/Rainer Unger (2001): Einkommen, Gesundheit und Mortalität in Deutschland, Großbritannien und den USA. In: Kölner Zeitschrift für Soziologie und Sozialpsychologie 53, S. 96-110.

Klein, Thomas/Rainer Unger/Alexander Schulze (2006): Bildungsexpansion und Lebenserwartung. In: Andreas Hadjar/Rolf Becker (Hrsg.), Die Bildungsexpansion. Erwartete und unerwartete Folgen. Wiesbaden: VS Verlag für Sozialwissenschaften, S. 311-331.

Klemm, Klaus (1991): Jugendliche ohne Ausbildung. Die „Kellerkinder“ der Bildungsexpansion. In: Zeitschrift für Pädagogik 37, S. 887-898.

Krause, Peter (1987): Lebensbedingungen und wahrgenommene Lebensqualität von Arbeitslosen. In: Hans-Jürgen Krupp/Ute Hanefeld (Hrsg.), Lebenslagen im Wandel. Analysen 1987. Frankfurt a.M./New York: Campus, S. 105-125.

Kreft, Ita/Jan de Leeuw (1998): Introducing Multilevel Modelling. London: Sage.

Lang, Sabine/Maria Müller-Andritzky (1984): Gesundheit und soziale Integration. In: Wolfgang Glatzer/Wolfgang Zapf (Hrsg.), Lebensqualität in der Bundesrepublik. Frankfurt a.M./New York: Campus, S. 141-156.

Langer, Wolfgang (2004): Mehrebenenanalyse. Eine Einführung für Forschung und Praxis. Wiesbaden: VS Verlag für Sozialwissenschaften.

Lindenberg, Siegwart/Bruno S. Frey (1993): Alternatives, Frames, and Relative Prices. A Broader View of Rational Choice Theory. In: Acta Sociologica 36, S. 191-205.

Markovsky, Barry (1987): Toward Multilevel Sociological Theories. Simulation of Actor and Network Effects. In: Sociological Theory 5, S. 101-117.

Mayer, Karl Ulrich (1994): Bildung und Arbeit in einer alternden Bevölkerung. In: Paul B. Baltes/Jürgen Mittelstraß/Ursula M. Staudinger (Hrsg.), Alter und Altern. Ein interdisziplinärer Studientext zur Gerontologie. Berlin: de Gruyter, S. 518-543.

Mayer, Karl Ulrich/Hans-Peter Blossfeld (1990): Die gesellschaftliche Konstruktion sozialer Ungleichheit im Lebensverlauf. In: Peter A. Berger/Stefan Hradil (Hrsg.), Lebenslagen - Lebensläufe - Lebensstile. Göttingen: Schwartz, S. 297-318.

Mayer, Karl Ulrich/Johannes Huinink (1990): Alters-, Perioden-, Kohorteneffekte in der Analyse von Lebensverläufen oder: Lexis ade? In: Karl Ulrich Mayer (Hrsg.), Lebensverläufe und sozialer Wandel. Sonderheft der Kölner Zeitschrift für Soziologie und Sozialpsychologie. Opladen: Westdeutscher Verlag, S. 442-459.

Mayring, Philipp A. (1991): Die Erfassung subjektiven Wohlbefindens. In: Andrea Abele/Peter Becker (Hrsg.), Wohlbefinden: Theorie, Empirie, Diagnostik. Weinheim/München: Juventa, S. 51-70. 
Mohr, Hans-Michael (1987): Ausgewählte Aspekte zur wahrgenommenen Lebensqualität. In: Hans-Jürgen Krupp/Ute Hanefeld (Hrsg.), Lebenslagen im Wandel. Analysen 1987. Frankfurt a.M./New York: Campus, S. 161-179.

Mueller, Ulrich/Monika Heinzel-Gutenbrunner (2001): Krankheiten und Beschwerden (subjektive Gesundheit) unter Bewertung der eigenen Gesundheit. Materialien zur Bevölkerungswissenschaft, Heft 102c. Wiesbaden: Bundesinstitut für Bevölkerungsforschung beim Statistischen Bundesamt.

Müller, Walter (1998): Erwartete und unerwartete Folgen der Bildungsexpansion. In: Jürgen Friedrichs/Rainer M. Lepsius/Karl Ulrich Mayer (Hrsg.), Die Diagnosefähigkeit der Soziologie. Opladen: Westdeutscher Verlag, S. 83-112.

Noll, Heinz-Herbert (1989): Indikatoren des subjektiven Wohlbefindens. Instrumente für die gesellschaftliche Dauerbeobachtung und Sozialberichterstattung? In: ZUMA-Nachrichten 24, S. 26-41.

Ormel, Johan/Siegwart Lindenberg/Nardi Steverink/Lois M. Verbrugge (1999): Subjective WellBeing and Social Production Functions. In: Social Indicators Research 46, S. 61-90.

Oswald, Andrew J. (1997): Happiness and Economic Performance. In: The Economic Journal 107 , S. $1815-1831$.

Plum, Wolfgang (1982): Kohortenanalyse von Umfragedaten. Zur Identifizierung möglicher Einflußfaktoren politischen Verhaltens und politischer Einstellungen. In: Kölner Zeitschrift für Soziologie und Sozialpsychologie 34, S. 509-532.

Rippl, Susanne/Dirk Baier/Angela Kindervater/Klaus Boehnke (2005): Die EU-Osterweiterung als Mobilisierungsschub für ethnozentrische Einstellungen? In: Zeitschrift für Soziologie 34, S. 288-310.

Robinson, William S. (1950): Ecological Correlations and the Behavior of Individuals. In: American Sociological Review 15, S. 351-357.

Runciman, Walter G. (1966): Relative Deprivation and Social Justice. London: Routledge and Kegan Paul.

Ryder, Norman B. (1965): The Cohort as a Concept in the Study of Social Change. In: American Sociological Review 30, S. 843-861.

Schelsky, Helmut (1956): Soziologische Bemerkungen zur Rolle der Schule in unserer Gesellschaftsverfassung. In: ders. (Hrsg.), Schule und Erziehung in der industriellen Gesellschaft. Würzburg: Weltbund Verlag, S. 9-50.

Schneider, Sven (1999): Das subjektive Gesundheitsempfinden im Lebensverlauf. In: Österreichische Zeitschrift für Soziologie 24, S. 47-62.

Schwarz, Norbert/Fritz Strack (1985): Cognitive and Affective Processes in Judgments of WellBeing. A Preliminary Model. In: Hermann Brandstädter/Erich Kirchler (Hrsg.), Economic Psychology. Linz: Trauner, S. 439-448.

Scott, Jacqueline/Jane Nolan (2005): Aging Positive? Not According to the British Public. GeNet Working Paper No. 7

Snijders, Tom A.B./Roel J. Bosker (1999): Multilevel Analysis. An Introduction to Basic and Advanced Multilevel Modelling. London: Sage.

Solga, Heike/Sandra Wagner (2001): Paradoxie der Bildungsexpansion. Die doppelte Benachteiligung von Hauptschülern. In: Zeitschrift für Erziehungswissenschaft 4, S. 107-127.

Spellerberg, Anette/Detlef Landua/Roland Habich (1992): Orientierungen und subjektives Wohlbefinden in West- und Ostdeutschland. In: Wolfgang Glatzer/Heinz-Herbert Noll (Hrsg.), Lebensverhältnisse in Deutschland. Ungleichheit und Angleichung. Soziale Indikatoren, Band XI. Frankfurt a.M./New York: Campus, S. 249-278.

Stephan, Walter G./Cookie W. Stephan (2000): An Integrated Threat Theory of Prejudice. In: Stuart Oskamp (Hrsg.), Reducing Prejudice and Discrimination. Mahwah: Erlbaum, S. 23-46. 
Terwey, Michael (1990): Zur Wahrnehmung von wirtschaftlichen Lagen in der Bundesrepublik. In: Walter Müller/Peter Ph. Mohler/Barbara Erbslöh/Martina Wasmer (Hrsg.), Blickpunkt Gesellschaft. Einstellungen und Verhalten der Bundesbürger. Opladen: Westdeutscher Verlag, S. 144-190.

Tuma, Nancy Brandon/Michael T. Hannan (1984): Social Dynamics. Models and Methods. Orlando: Academic Press.

Veenhoven, Ruut (1995): The Cross-national Pattern of Happiness. Test of Predictions Implied in Three Theories of Happiness. In: Social Indicators Research 34, S. 33-68.

Veenhoven, Ruut (1997): Die Lebenszufriedenheit der Bürger. Ein Indikator für die „Lebbarkeit“ von Gesellschaften? In: Heinz-Herbert Noll (Hrsg.), Sozialberichterstattung in Deutschland. Konzepte, Methoden und Ergebnisse für Lebensbereiche und Bevölkerungsgruppen. Weinheim/München: Juventa, S. 267-293.

Veenhoven, Ruut (2001): Happiness in Society. In: Jutta Allmendinger (Hrsg.), Gute Gesellschaft? Verhandlungen des 30. Kongresses der Deutschen Gesellschaft für Soziologie in Köln 2000. Opladen: Leske + Budrich, S. 1265-1314.

Wagner, Michael (2001): Kohortenstudien in Deutschland. Expertise für die Kommission zur Verbesserung der informationellen Infrastruktur zwischen Wissenschaft und Statistik. Köln: Universität zu Köln.

Wilson, Warner (1967): Correlates of Avowed Happiness. In: Psychological Bulletin 67, S. 294-306.

Winkelmann, Liliana/Rainer Winkelmann (1998): Why are Unemployed so Unhappy? Evidence from Panel Data. In: Economica 65, S. 1-15.

Zapf, Wolfgang/Jürgen Schupp/Roland Habich (Hrsg.) (1996): Lebenslagen im Wandel: Sozialberichterstattung im Längsschnitt. Frankfurt a.M./New York: Campus.

\section{Andreas Hadjar}

Geb. 1974. Dr. phil., Oberassistent in der Abteilung Bildungssoziologie an der Universität Bern. Forschungsschwerpunkte: Politische Soziologie, Methoden der empirischen Sozialforschung und Datenanalyseverfahren, Familienforschung, Bildungssoziologie. Ausgewählte Veröffentlichungen: Ellenbogenmentalität und Fremdenfeindlichkeit bei Jugendlichen. Die Rolle des Hierarchischen Selbstinteresses, 2004; (mit R. Becker) (Hrsg.) Die Bildungsexpansion. Erwartete und unerwartete Folgen, 2006; (mit D. Baier/K. Boehnke/J. Hagan) Juvenile Delinquency and Gender Revisited: The Family and Power-Control Theory Reconceived. In: European Journal of Criminology 4, 2007.

\section{Sigrid Haunberger}

Geb. 1975. Dipl. Soz., wissenschaftliche Assistentin in der Abteilung Bildungssoziologie der Universität Bern. Forschungsschwerpunkte: Bildungssoziologie, Soziologie der Arbeit, Soziologie der Kindheit, Methoden und Techniken empirischer Sozialforschung, Interviewereffekte. Ausgewählte Veröffentlichungen: Interviewer und Befragte im Kinderpanel. Interviewdauer und Panelbereitschaft. In: C. Alt (Hrsg.): Kinderleben - Aufwachsen zwischen Familie, Freunden und Institutionen. Band 2: Aufwachsen zwischen Freunden und Institutionen, 2005; Das standardisierte Interview als soziale Interaktion: Interviewereffekte in der Umfrageforschung, ZA-Information $58,2006$.

\section{Frank Schubert}

Geb. 1975. Dipl. Soz., wissenschaftlicher Assistent in der Abteilung Bildungssoziologie an der Universität Bern. Forschungsschwerpunkte: International vergleichende Bildungsforschung, Übertritt vom Bildungssystem in den Arbeitsmarkt, Methoden der empirischen Sozialforschung. Ausgewählte Veröffentlichungen: (mit R. Becker) Soziale Ungleichheiten von Lesekompetenzen. Eine Matching-Analyse im Längsschnitt mit Querschnittsdaten von PIRLS 2001 und PISA 2000. 
In: Kölner Zeitschrift für Soziologie und Sozialpsychologie, Heft 2, 2006; (mit I. Kogan) General Indicators on Transition from School to Work. Youth Transitions from Education to Working Life in Europe, Part I. In: Statistics in Focus, Heft 4, 2003; (mit I. Kogan) Youth Transitions from Education to Working Life in Europe: A General Overview. In: I. Kogan/W. Müller (Hrsg.): School-to-Work Transitions in Europe: Analyses of the EU LFS 2000 Ad Hoc Module, 2003. 